Draft VERSion December 17, 2013

Preprint typeset using $\mathrm{L}^{A} \mathrm{~T}_{\mathrm{E}} \mathrm{X}$ style emulateapj v. 11/10/09

\title{
NGC 2207/IC 2163: A GRAZING ENCOUNTER WITH LARGE SCALE SHOCKS
}

\author{
Michele Kaufman $^{1}$, Dirk Grupe ${ }^{2}$, Debra M. Elmegreen ${ }^{3}$, Bruce G. Elmegreen $^{4}$, Curtis Struck $^{5}$, Elias Brinks ${ }^{6}$ \\ Draft version December 17, 2013
}

\begin{abstract}
Radio continuum, Spitzer infrared, optical, and XMM-Newton X-ray and ultraviolet observations $(U V W 1$ and $U V M 2$ ) are used to study large-scale shock fronts, young star complexes, and the galactic nuclei in the interacting galaxies NGC 2207/IC 2163. There are two types of large-scale shock fronts in this galaxy pair. The large-scale shock front along the rim of the ocular oval in IC 2163 has produced vigorous star formation in a dusty environment, bright in the Spitzer $8 \mu \mathrm{m}$ and $24 \mu \mathrm{m}$ images. This large-scale shock lies behind NGC 2207 and is not prominent in X-rays. In the outer part of the companion side of NGC 2207, a large-scale front attributed to disk or halo scraping is particularly bright in the $\lambda 6 \mathrm{~cm}$ and $\lambda 20 \mathrm{~cm}$ radio continuum but not in any tracers of recent star formation ( $\mathrm{H} \alpha, 8 \mu \mathrm{m}, 24 \mu \mathrm{m}$, or ultraviolet emission) or in X-rays. This radio-continuum front is simply due to compression of the magnetic field and may be mainly in the halo on the back side of NGC 2207, between the two galaxies. Values of the ratio of $8 \mu \mathrm{m}$ to $\lambda 6 \mathrm{~cm}$ radio continuum flux density of prominent, kpc-sized, Spitzer IRAC star-forming clumps in NGC 2207/IC 2163 are compared with those of giant radio H II regions in M81. For the bright clumps in NGC 2207, the mean value of this ratio is the same as for the M81 H II regions, whereas for the bright clumps on the rim of the IC 2163 ocular oval, the mean value is nearly a factor of two greater. On the other hand, the galaxy pair has global values of the ratios of infrared to radio continuum flux density in the Spitzer $8 \mu \mathrm{m}, 24 \mu \mathrm{m}$, and $70 \mu \mathrm{m}$ bands and the IRAS FIR significantly below the medians/means for large samples of galaxies. Feature i, a mini-starburst on an outer arm of NGC 2207 on its anti-companion side, is the most luminous $8 \mu \mathrm{m}, 24 \mu \mathrm{m}, 70 \mu \mathrm{m}$, radio continuum, and $\mathrm{H} \alpha$ source in the galaxy pair. Highly absorbed, it is not detected in X-rays and is fainter in the ultraviolet than the clump containing SN 1999ec, $8^{\prime \prime} \mathrm{SSE}$ of the core of feature $i$. We find evidence that a radio supernova was present in the core of feature $i$ in 2001. X-ray emission is detected from the nucleus of NGC 2207 and from nine discrete sources whose X-ray luminosities make them possible candidates for ULXs. One of these corresponds with the Type Ib SN 1999ec, and another may be a radio supernova or a background quasar. The X-ray luminosity of the NGC 2207 nucleus is $\log L_{0.3-10.0 \mathrm{keV}}=40.6\left[\mathrm{ergs} \mathrm{s}^{-1}\right]$, which, together with its X-ray spectrum, suggests that this is a highly absorbed, low-luminosity, Active Galactic Nucleus. Subject headings: galaxies: interactions, galaxies: individual (NGC 2207/IC 2163), radio continuum: galaxies, supernovae: individual (SN 1999ec), X-rays: galaxies
\end{abstract}

\section{INTRODUCTION}

The spiral galaxies NGC 2207 and IC 2163 at a distance of $35 \mathrm{Mpc}\left(1^{\prime \prime}=170 \mathrm{pc}\right)$ (Elmegreen et al. 1995a) and partially overlapping in projection are involved in a nearly-grazing encounter with closest approach 200 - 400 Myr ago. We have studied this pair extensively (Elmegreen et al. 1995a, b, 1998, 2000, 2001, 2006; Thomasson 2004; Struck et al. 2005) throughout the electromagnetic spectrum with $H S T$ WFPC2 observations in $U B V I$ bands, ground-based $\mathrm{H} \alpha$, Spitzer IRAC

\footnotetext{
${ }^{1}$ Department of Physics, The Ohio State University, 191 West Woodruff Ave, Columbus, OH 43210; email: rallis@mps.ohiostate.edu

${ }^{2}$ Department of Astronomy and Astrophysics, Pennsylvania State University, 525 Davey Lab, University Park, PA 16802; email: grupe@astro.psu.edu

${ }^{3}$ Department of Physics and Astronomy, Vassar College, 124 Raymond Av., Poughkeepsie, NY 12604; email: elmegreen@vassar.edu

${ }^{4}$ IBM Research Division, T.J. Watson Research Center, PO Box 218, Yorktown Heights, NY 10598; email: bge@us.ibm.com

${ }^{5}$ Department of Physics and Astronomy, Iowa State University, Ames, IA 50011; email: curt@iastate.edu

'University of Hertfordshire, Centre for Astrophysics Research, College Lane, Hatfield AL10 9AB, UK; email: E.Brinks@herts.ac.uk
}

$(3.6-8 \mu \mathrm{m})$ and MIPS $(24-160 \mu \mathrm{m})$ observations, VLA $\mathrm{H} \mathrm{I}$ and radio continuum, and ${ }^{12} \mathrm{CO} J=1 \rightarrow 0$ (SEST) observations and reproduced many of the observed features with $\mathrm{N}$-body and SPH encounter simulations. Relative to IC 2163, the encounter is prograde and nearly inplane, producing the observed eye-shaped (ocular) oval and two long tidal arms in IC 2163. Relative to NGC 2207, the encounter is retrograde with IC 2163 moving behind NGC 2207 towards the east. The short-lived ocular phase and other features of this system set strict constraints on the numerical model for the encounter. Along the rim of the ocular oval there is a large-scale shock front caused by the inflow of gas responding to tidal torques. This observed shock is a signature of the early stages of prograde grazing encounters. The models in Struck et al. (2005) predict that disk or halo scraping between the companion sides of the two galaxies would push shocks at a few hundred $\mathrm{km} \mathrm{s}^{-1}$ into each other across a front $30^{\prime \prime}$ to $60^{\prime \prime}$ in length, with a mass transfer stream from IC 2163 impinging on NGC 2207. Evidence for this is seen in the radio continuum image as enhanced radio emission from the outer part of the companion sides of NGC 2207 and IC 2163. According to the models in Struck et al. (2005), the two galaxies in this system will 
eventually merge.

To extend our previous studies of this galaxy pair, we observed NGC 2207/IC 2163 in X-rays and the ultraviolet with $X M M-N$ ewton and made new radio continuum observations with the VLA 7 at $\lambda 6 \mathrm{~cm}$ at a resolution of $2.5^{\prime \prime}$, comparable to that of the ultraviolet and Spitzer IRAC images. The goals of the X-ray observations were (1) to detect the predicted soft X-ray emission from diffuse hot plasma at the large-scale shock fronts produced by the grazing encounter and (2) to determine the number, location, and nature of bright, discrete $\mathrm{X}$ ray sources in this pair. The XMM-Newton observations presented here are the first deep X-ray observations of NGC 2207/IC 2163. Note that this galaxy pair was not detected in the ROSAT All-Sky Survey (RASS; Voges et al. 1999).

We use our radio continuum, Spitzer infrared, optical, and XMM-Newton X-ray, $U V W 1$ (effective $\lambda=2910$ $\AA$ ) and $U V M 2$ (effective $\lambda=2310 \AA$ ) observations to study the large-scale shock fronts, the young star complexes, the NGC 2207 nucleus, and various discrete X-ray sources in these galaxies. In an outer spiral arm on the anti-companion side of NGC 2207, there is a morphologically peculiar star-forming region (called feature $i$ by Elmegreen et al. 2000) which is the most luminous $\mathrm{H} \alpha$, radio continuum, $8 \mu \mathrm{m}, 24 \mu \mathrm{m}$, and $70 \mu \mathrm{m}$ source in NGC $2207 /$ IC 2163. At $24 \mu \mathrm{m}$, it accounts for $\simeq 12 \%$ (Elmegreen et al. 2006) of the total emission from the galaxy pair. Feature $i$ contains an opaque dust cone (400 $\mathrm{pc}$ in projected length) aligned nearly parallel to the minor axis of the projection of NGC 2207 into the sky plane. We present new results on feature $i$ and its environs.

With a star formation rate (SFR) deduced from $\mathrm{H} \alpha$ emission, IC 2163 and NGC 2207 each have a $\mathrm{SFR} / M(\mathrm{HI})$ typical of normal spiral disks. The radio continuum flux density of NGC $2207 /$ IC 2163 is about 3 times higher than expected from the IRAS far-infrared flux (Elmegreen et al. 1995a), yet neither galaxy contains a radio-loud AGN. The global value of the Helou $q_{\text {FIR }}$ parameter (the logarithm of the ratio of FIR to $\lambda 20 \mathrm{~cm}$ radio continuum flux density) is 1.81 for NGC $2207 / \mathrm{IC}$ 2163, whereas Condon (1992) finds the median value of $q_{\text {FIR }}$ for galaxies that are not radio-loud AGNs is $\simeq 2.3$ with an rms scatter of $\leq 0.2$. Explaining why the radio continuum emission from the galaxy pair is enhanced without a commensurate effect on star formation is important for understanding the conditions necessary for star formation in general. Some other galaxies with a similarly low global value of the Helou $q_{\mathrm{FIR}}$ parameter are NGC 2276 (Hummel \& Beck 1995) and the Taffy pairs UGC 12914/15 and UGC 813/6 (Condon et al. 1993, 2002). Like NGC 2207, the spiral galaxy NGC 2276 has enhanced radio emission in the outer part of the disk on one side of the galaxy. NGC 2207 and NGC 2276 have about the same redshift, radio continuum flux density and $B_{T}$. Rasmussen et al. (2006) observed the interacting galaxy pair NGC 2276/NGC 2300 with Chandra and found a shock-like feature in X-rays. NGC 2276 had already been observed in X-rays with the ROSAT High-

\footnotetext{
7 The National Radio Astronomy Observatory is a facility of the National Science Foundation operated under cooperative agreement by Associated Universities, Inc. The observations are from VLA Programs AK 368 and AK 526.
}

Resolution Imager (Davis et al. 1997). We shall compare NGC 2207/IC 2163 with NGC 2276.

Section 2 describes our new observations (X-ray and ultraviolet from $X M M-N e w t o n$ and $\lambda 6 \mathrm{~cm}$ radio continuum from the VLA) and the data reductions. Section 3 presents an overview of the system. Section 4 discusses star-forming clumps prominent in the Spitzer $8 \mu \mathrm{m}$ (IRAC 4) image and/or in the $X M M$-Newton ultraviolet images $(U V M 2$ and $U V W 1)$. Values of the flux density ratio $\mathrm{S}_{\nu}(8 \mu \mathrm{m}) / \mathrm{S}_{\nu}(\lambda 6 \mathrm{~cm})$ for these $\mathrm{kpc}$-sized clumps are compared with those of giant HII regions in M81 as an example of what is normal for an OB association. Section 5 presents our results on the large-scale shock fronts and comments on infrared to radio continuum ratios. Section 6 describes our X-ray results. Section 7 compares NGC 2207 with NGC 2276. Section 8 is devoted to feature $i$ and its environs. Section 9 summarizes our conclusions.

For this galaxy pair, we adopt the distance of $35 \mathrm{Mpc}$ as in Elmegreen et al. (1995a), who used $H_{0}=75 \mathrm{kpc}$ $\mathrm{s}^{-1} \mathrm{Mpc}^{-1}$.

\section{OBSERVATIONS AND DATA REDUCTION}

\subsection{XMM-Newton observations}

XMM-Newton observed NGC 2207/IC 2163 on 2005 August 31 for a total of $51.6 \mathrm{ks}$. A summary of the observations in each of the instruments onboard $X M M$ Newton is given in Table[1. The European Photon Imaging Camera (EPIC) pn (Strüder et al. 2001) was operated in Extended Full-Frame mode and the two EPIC MOS (Turner et al. 2001) in Full-Frame mode. All observations with the EPICs were performed with the thin filters. Due to some episodes of high particle background at the beginning of the observations, part of the pn observation had to be discarded leaving a net observing time of $34.2 \mathrm{ks}$. The MOS data, however, were not affected by this high background flux and we used the entire observations.

The XMM-Newton data were analyzed in the standard way using the XMMSAS version $x m m$ sas_20060628_1801-7.0.0. Only single and double events (PATTERN.le.4) and single to quadruple events (PATTERN.le.12) were selected for the pn and MOS data, respectively. Events in or next to the CCD gaps were rejected from the analysis (FLAG.eq.0). For our final X-ray image, the screened event files of the pn and MOS data were merged with the XMMSAS task merge. The spectra were rebinned by grppha version 3.0.0 with 20 photons per bin in the pn and 15 counts per bin in the two MOS cameras. The redistribution matrices and the auxiliary response files were created by the XMMSAS tasks rmfgen and arfgen, respectively. Spectral fits to the EPIC pn and MOS spectra were performed with XSPEC version 12.5.0ac (Arnaud 1996). All errors are $90 \%$ confidence unless stated otherwise.

Throughout the paper spectral indexes are denoted as energy spectral indexes with flux density $F_{\nu} \propto \nu^{-\alpha}$. Spectral index has the same type of definition for the $\mathrm{X}$-ray and for the radio observations

For the MOS X-ray image, the FWHM of the pointspread function (PSF) is $\sim 5^{\prime \prime}$ on-axis. The $4.1^{\prime \prime}$ pixel size for the pn X-ray camera results in somewhat poorer spatial resolution than for the MOS cameras $\left(1.1^{\prime \prime}\right.$ pixels). According to the XMM-Newton User's Handbook, 
the core of the PSF for the X-ray cameras varies little over the energy range $0.1-4 \mathrm{keV}$ and is somewhat triangular in shape for the MOS2 camera. The half energy width (at which $50 \%$ of the total energy is encircled) is $\sim 15^{\prime \prime}$ for the XMM-Newton X-ray images.

Comparison of the XMM-Newton X-ray, radio continuum, and Spitzer infrared positions of the NGC $2207 \mathrm{nu}$ cleus indicates that the positional accuracy of the X-ray data is about $3^{\prime \prime}$.

We also took advantage of XMM-Newton's multiwavelength capacity by using the Optical Monitor (OM; Mason et al. 2001) performing photometry in 4 filters $(B$, $U, U V W 1$, and $U V M 2$ ). We use the $U V W 1$ (effective $\lambda=2910 \AA$ ) and $U V M 2$ (effective $\lambda=2310 \AA$ ) images to study prominent star-forming clumps by comparing the ultraviolet, radio continuum, and $8 \mu \mathrm{m}$ flux densities and $\mathrm{H} \alpha$ fluxes. The $U V M 2$-band, with response to the $\lambda$ range $2000 \AA$ to $2700 \AA$, is somewhat similar to the GALEX NUV, which has response to the $\lambda$ range $1750 \AA$ to $2750 \AA$ with an effective wavelength of $2267 \AA$. The observing times and exposure times are listed in Table1. The OM data were processed with the XMMSAS task omichain. During the course of the observations a $3^{\prime \prime}$ southward drift in declination occurred and the OM onboard software did not correct for it. Instead, by using foreground stars in the Guide Star Catalog or in the 2MASS image or in the Spitzer IRAC $1(3.6 \mu \mathrm{m})$ image as standard stars, we applied a plate solution to the ultraviolet images to register them to the same coordinates as the Spitzer and radio continuum images. The task omichain creates for each exposure a source list containing raw and corrected counts $\mathrm{s}^{-1}$ and magnitudes. We used the source-list data on foreground stars in the field outside of the galaxies to convert image units to counts $\mathrm{s}^{-1}$ and magnitudes in the final stacked image and to check on corrections for dead time and sensitivity degradation.

\subsection{Radio observations at $\lambda 6 \mathrm{~cm}$}

With the VLA we observed NGC $2207 /$ IC 2163 in the radio continuum at a central frequency of $4860.1 \mathrm{MHz}$ for 92 minutes (on the target) in B configuration on 2001 April 14 and for 50 minutes (on the target) in D configuration on 1995 May 13. The observations were made with one IF pair at $4885.1 \mathrm{MHz}$ with a $50 \mathrm{MHz}$ bandwidth and the other at $4835.1 \mathrm{MHz}$ with a $50 \mathrm{MHz}$ bandwidth. The phase center was RA, Dec $(2000)=06$ $1622.665,-212206.87$. For the B configuration (high resolution) observations, the phase calibrator was 0606223, the flux calibrators were 3C 286 and 3C 147, and the polarization calibrators were $3 \mathrm{C} 138$ and $3 \mathrm{C} 286$. No significant polarization was detected. For the D configuration (low resolution) observations, the phase calibrator was 0607-157 and the flux standard was 3C 286. Our D configuration observations were not appropriate for a polarization calibration.

The AIPS software package was used for the data reduction. After calibrating the uv data from each of the VLA configurations separately and checking the separate maps, we combined the uv data sets from the two configurations and ran the AIPS task IMAGR with ROBUST $=-2$ to make and clean a map with a synthesized beam of $2.48^{\prime \prime} \times 1.3^{\prime \prime}(\mathrm{HPBW}), \mathrm{BPA}=8^{\circ}$. After con- volution to a circular beam of $2.5^{\prime \prime}$ (HPBW) and correction for primary beam attenuation, this became our final $\lambda 6 \mathrm{~cm}$ high resolution image. In the field of interest, the maximum correction for primary beam attenuation was a factor of 1.2. In this image, which is displayed in Figure1, a surface brightness of $1 \mathrm{mJy}^{\mathrm{beam}}{ }^{-1}$ corresponds to $T_{b}=8.279 \mathrm{~K}$ and the rms noise is 0.016 mJy beam $^{-1}$, equivalent to $T_{b}=0.13 \mathrm{~K}$. We find a total flux density from the galaxy pair in this image $\mathrm{S}_{\nu}(4.86$ $\mathrm{GHz})=0.132 \pm 0.001 \mathrm{Jy}$, with about $20 \%$ of this from IC 2163. The single-dish observations of the Parkes-MITNRAO survey(Griffith et al. 1994) list $\mathrm{S}_{\nu}(4.85 \mathrm{GHz})=$ $0.10 \pm 0.01 \mathrm{Jy}$ for NGC 2207; it is not clear whether the latter includes IC 2163.

\subsection{Additional Data}

Other images of this galaxy pair that we use here are the WFPC2 HST B-band image from Elmegreen et al. (2000), the Spitzer IRAC and MIPS images from Elmegreen et al. (2006), the $\mathrm{H} \alpha$ image from Elmegreen et al. (2001), the VLA H I and line-free $\lambda 20 \mathrm{~cm}$ radio continuum images from Elmegreen et al. (1995a), and a radio continuum image at $8.46 \mathrm{GHz}(\lambda 3.5$ $\mathrm{cm}$ ) from the VLA public archives (Program AK 509) from 2003 January 14 observations. Table2 lists the FWHM of the point-spread functions (PSF) of the images we use. The VLA $\mathrm{H}$ I and radio continuum images have Gaussian synthesized beams (point-spread functions). The other images do not, and some of the nonradio images, such as the Spitzer $8 \mu \mathrm{m}$ and $24 \mu \mathrm{m}$ images and the XMM-Newton X-ray images, have significant sidelobes.

\section{OVERVIEW OF THE SYSTEM}

Figure1 displays our $\lambda 6 \mathrm{~cm}$ radio continuum image and the WFPC2 HST B-band image of this galaxy pair, and Figure 2 displays our $U V M 2$ image and the Spitzer $8 \mu \mathrm{m}$ image overlaid with contours of the line-of-sight $\mathrm{H} \mathrm{I}$ column density $N(\mathrm{HI})$ associated with each galaxy. The two galaxies partially overlap in projection, with NGC 2207 in front. IC 2163 has an eye-shaped oval midway out in the disk and a tidal tail on the anti-companion side. The H I image of IC 2163 reveals a symmetric tidal bridge arm on the companion side; it is harder to discern in the infrared or optical because it lies behind the central disk of NGC 2207 but can be faintly traced in the HST B-band (see Fig. 3 in Elmegreen et al. 2001) and Spitzer IRAC images. The rim of the eye-shaped oval (which we call the eyelids) in IC 2163 is outlined by optical, radio continuum, $\mathrm{H}$ I and infrared emission and is particularly bright in the Spitzer $8 \mu \mathrm{m}$ and $24 \mu \mathrm{m}$ images. The eyelids are one of the large scale shock fronts that we investigate in Section 5. They are produced by radial streaming and convergence of orbits due to tidal forces (see the velocity vectors of the model displayed in Fig. 2 of Elmegreen et al. 2000), which concentrate old stars as well as young stars in the eyelids (Elmegreen et al. 1995a). The other large scale shock is the long ridge of enhanced radio continuum emission on the companion (eastern) side of NGC 2207; in Figure1 a tilted box 54" $(9 \mathrm{kpc})$ long is drawn around it. We call it the $N E$ radio

8 Image credit: NRAO/VLA Archive Survey, (c) 2005-2007 AUI/NRAO 
ridge. Aside from the highly luminous feature $i$, the companion side of NGC 2207 is substantially brighter in the radio continuum than its anticompanion side, with the brightest large-scale radio emission coming from the $N E$ radio ridge. The radio continuum emission from the adjacent companion side of IC 2163 is also enhanced. This is evidence of either disk or halo scraping between the two galaxies.

The $N E$ radio ridge contains optical spiral arms of NGC 2207 visible in the HST B-band image. These are not the outermost spiral arms of NGC 2207 on the companion side. The bottom panel in Figure 2 displays an outer H I arm of NGC 2207 cutting in front of the western part of the northern eyelid of IC 2163 and the eastern part of the southern eyelid. The extinction due to this outer arm of NGC 2207, which is seen backlit by IC 2163 in the HST image (Elmegreen et al. 2000), partly explains the faintness of the western part of the northern eyelid and the eastern part of the southern eyelid in the $U V M 2$ image. This arm has values of $\mathrm{N}(\mathrm{HI}) \geq 3 \times 10^{21}$ atoms $\mathrm{cm}^{-2}$. Assuming the outer disk of NGC 2207 is somewhat metal poor, Elmegreen et al. (2001) adopt for the relation between extinction and $\mathrm{H}$ I column density, $A_{v}=(0.35 \pm 0.18) \times 10^{-21} N(\mathrm{HI})$. For a foreground dust screen, the ultraviolet extinction $A(U V M 2)=2.6 A_{v}$, and $A(U V W 1)=2.0 A_{v}$ (Savage \& Mathis 1979). Thus $A(U V M 2)$ on this arm $\geq 2.7 \pm 1.4 \mathrm{mag}$. In the $\lambda 6 \mathrm{~cm}$ image in Figure1, another outer arm of NGC 2207 is visible cutting across just north of the nucleus of IC 2163.

There is also significant gas in the eyelids of IC 2163 . The top panel of Figure2 displays the N(HI) associated with IC 2163. Using the Swedish ESO Submillimeter Telescope (SEST), Thomasson (2004) detected ${ }^{12} \mathrm{CO} J=1 \rightarrow 0$ emission from both disks; the brightest ${ }^{12} \mathrm{CO} J=1 \rightarrow 0$ emission is from the central part of IC 2163 and has an integrated intensity, averaged over the beam, of $6 \mathrm{~K} \mathrm{~km} \mathrm{~s}^{-1}$, equivalent to $10^{21}$ $\mathrm{H}_{2} \mathrm{~cm}^{-2}$ if we use the Milky Way conversion factor $X_{\mathrm{CO}}=N\left(\mathrm{H}_{2}\right) / I_{\mathrm{CO}}=1.8 \pm 0.3 \times 10^{20}$ from Dame et al. (2001). The resolution of SEST (43" HPBW) is too low to tell if this ${ }^{12} \mathrm{CO} J=1 \rightarrow 0$ emission is mainly from the eyelids although the double-peaked nature of the ${ }^{12} \mathrm{CO}$ $J=1 \rightarrow 0$ line profile of IC 2163 suggests this may be the case (Struck et al. 2005). Except for a bright ${ }^{12} \mathrm{CO}$ $J=1 \rightarrow 0$ source on the northwest inner arm of NGC 2207 , the SEST ${ }^{12} \mathrm{CO} J=1 \rightarrow 0$ image closely resembles the Spitzer MIPS image at $160 \mu \mathrm{m}$ of this galaxy pair (Elmegreen et al. 2006). Both have about the same resolution, and the close correspondence tells us that the cooler dust measured by the $160 \mu \mathrm{m}$ emission has about the same distribution globally as the molecular gas, which is not surprising. We can infer the distribution of gas from the distributions of cooler and warmer dust, as indexed by the $70 \mu \mathrm{m}$ and $24 \mu \mathrm{m}$ emission, respectively. Aside from feature $i$, the brightest $70 \mu \mathrm{m}$ and $24 \mu \mathrm{m}$ emission in this galaxy pair is from the eyelids (see the figure in Section 5.1 below and the HiRes deconvolution of the $70 \mu \mathrm{m}$ and $24 \mu \mathrm{m}$ images in Velusamy et al. 2008), and thus the highest concentration of gas in IC 2163 is in the eyelids.

Elmegreen et al. (1995a) identified 11 unusually massive $\left(10^{8}-10^{9} M_{\odot}\right)$ H I clouds in NGC $2207 /$ IC 2163. Most of these are not sites of active star formation.
Clouds N1, N5, and N6 (three of the six massive H I clouds associated with NGC 2207) are labelled in Figure2. The center of H I Cloud N6 is at RA, Dec (2000) $=061625.560,-212219.08$. The brightest $\lambda 6 \mathrm{~cm}$ radio continuum source on the $N E$ radio ridge and the prominent Spitzer infrared clump IR 12 (see Section 4) are $3^{\prime \prime}$ west, $1^{\prime \prime}$ south of the center of H I cloud N6; SN $2003 \mathrm{H}$ is $2^{\prime \prime}$ east, $5^{\prime \prime}$ south of the center of $\mathrm{H}$ I Cloud N6. H I cloud N5 obscures the ultraviolet emission from the eastern part of the southern eyelid of IC 2163. At the center of Cloud $\mathrm{N} 5, \mathrm{~N}(\mathrm{HI})=4.8 \times 10^{21}$ atoms cm$^{-2}$, which corresponds to an $\mathrm{A}(U V M 2)$ of $4.4 \pm 2.2 \mathrm{mag}$. Elmegreen et al. (1993) discuss the formation of massive H I clouds and tidal dwarf galaxies by large-scale gravitational instabilities in the gas and suggest that $\mathrm{H}$ I Cloud N1 in the outer part of NGC 2207 where large $z$ motions are creating a warp may be in the process of forming a tidal dwarf galaxy. The only stellar emission detected from Cloud N1 forms a bow-shaped arc in the northwestern part of the cloud visible, for example, in the Digitized Sky Survey image, in the blue-band plate in Elmegreen et al. (1995a), and in the $U V M 2$ image in Figure 2 and Figure 3. It appears that star formation has commenced in only this part of Cloud N1.

\section{STAR-FORMING CLUMPS}

Figure3 displays the UVM2 image overlaid with Spitzer $8 \mu \mathrm{m}$ contours and with $\mathrm{H} \alpha$ contours, respectively. Aside from the effects of extinction, this demonstrates the generally good correspondence between these three tracers of recent star formation. We use photometry in $\lambda 6 \mathrm{~cm}$ radio continuum, $8 \mu \mathrm{m}, U V M 2, U V W 1$, and $\mathrm{H} \alpha$ bands to study star-forming clumps in this galaxy pair at a resolution of $2.0^{\prime \prime}-2.5^{\prime \prime}(0.3$ to $0.4 \mathrm{kpc})$.

Elmegreen et al. (2006) did photometry in the Spitzer IRAC bands of 225 bright clumps in this galaxy pair by using phot in IRAF with an aperture radius of $3.6^{\prime \prime}=0.61$ $\mathrm{kpc}$, and a local background annulus with inner radius $=$ $9.6^{\prime \prime}$ and outer radius $=15.6^{\prime \prime}$ concentric with the source aperture. The clumps are star complexes. In general, the source aperture includes collections of $\mathrm{OB}$ associations and older star clusters (see examples in Elmegreen et al. 2006). After registering the $8 \mu \mathrm{m}$ and ultraviolet images to the same coordinate grid as the high resolution $\lambda 6 \mathrm{~cm}$ image, we chose 28 prominent clumps in the $8 \mu \mathrm{m}$ and/or $U V M 2$ images; these are labelled in Figure 4, which displays the $8 \mu \mathrm{m}$ emission. For each clump, Table 3 lists the $8 \mu \mathrm{m}$ flux density from Elmegreen et al. (2006), the $\mathrm{H} \alpha$ flux from Elmegreen et al. (2001), and the $\lambda 6 \mathrm{~cm}$ flux density, $U V M 2$ magnitude, and $U V M 2-U V W 1$ color measured with the same choice of source aperture and local background annulus as for the IRAC measurements. The uncertainty in the $\lambda 6 \mathrm{~cm}$ flux density of each clump is 0.04 to $0.05 \mathrm{mJy}$. In addition to free-free radio emission from the $\mathrm{H}$ II regions, the source aperture is likely to include nonthermal radio emission from the spiral arms, some of which is removed by the local background subtraction. For $U V M 2$ and $U V W 1$ we took from the XMM-Newton User's Handbook the zero-points for the magnitudes (defined such that Vega $=0.025 \mathrm{mag}$ ) and the conversion factors to get from the count rates to flux densities in mJy. The conversion factors are for white dwarfs and thus the values of the $U V M 2$ flux density used in Table 3 for the ratio of $8 \mu \mathrm{m}$ to $U V M 2$ flux 
density are rough estimates.

The numbering of the clumps is the same as in Elmegreen et al. (2006) except for the added clumps $u 1$ at RA, Dec $(2000)=061620.298,-212226.47$ and $r c 1$ at RA, Dec $(2000)=061617.996,-2122$ 04.16. In section 6 , we find that clumps IR 11 , IR 21, and rc1 coincide with discrete X-ray sources. The first eleven clumps in Table 3 are in IC 2163; the rest are in NGC 2207.

The values of $A_{v}$ in Table 3 for the NGC 2207/IC 2163 clumps are upper limits obtained from the $\lambda 6 \mathrm{~cm}$ flux density and $\mathrm{H} \alpha$ flux for case $\mathrm{B}$ recombination with $T_{e}=10^{4} \mathrm{~K}$ by assuming all of the $\lambda 6 \mathrm{~cm}$ flux density after subtracting the local background is optically-thin free-free emission. For a number of the clumps the $A_{v}$ upper limits are quite large; this leads us to suspect that some clumps include significant nonthermal radio emission at $\lambda 6 \mathrm{~cm}$. Within a given clump, the extinction is far from uniform as the $H S T$ observations found lots of dust features on very small spatial scales near star clusters whose blue colors indicate little extinction (Elmegreen et al. 2001).

As an example of what is expected for the flux density ratio $S_{\nu}(8 \mu \mathrm{m}) / S_{\nu}(6 \mathrm{~cm})$ of an OB association, we present data in Table 4 on the giant $\mathrm{H}$ II regions in M81. We chose the 11 giant radio $H$ II regions in M81 which have the highest signal-to-noise in the $\lambda 6 \mathrm{~cm}$ radio continuum observations of Kaufman et al. (1987) and a radio spectral index $\alpha$ consistent with optically-thin free-free emission. For these M81 H II regions, Table 4 uses the $\lambda 6 \mathrm{~cm}$ radio continuum flux densities from Kaufman et al. (1987) and the Spitzer $8 \mu \mathrm{m}, 24 \mu \mathrm{m}$, and GALEX NUV flux densities from Pérez-González et al. (2006) and finds the mean value of the ratio $S_{\nu}(8 \mu \mathrm{m}) / S_{\nu}(6 \mathrm{~cm})=19$ with the standard deviation $\sigma$ of the sample $=5$, and the mean value of the ratio $S_{\nu}(24 \mu \mathrm{m}) / S_{\nu}(6 \mathrm{~cm})=36$ with $\sigma=9$. (The average measurement uncertainties are \pm 3 and \pm 5 , respectively). Comparison of these ratios with global values for entire galaxies is given in Section 5.3. The M81 $\mathrm{H}$ II regions have relatively low extinction. Two estimates of the extinction $A_{v}$ are listed for each $\mathrm{H}$ II region: $A_{v}(\mathrm{~K})$ from Kaufman et al. (1987) is derived from $S_{\nu}(6$ $\mathrm{cm}) / \mathrm{S}(\mathrm{H} \alpha) ; A_{v}(\mathrm{PG})$ from Pérez-González et al. (2006) is obtained from the line ratios $\mathrm{H} \alpha / \mathrm{H} \beta$ and $\mathrm{H} \alpha / \mathrm{Pa} \alpha$. Except for one H II region, these two methods give the same values for $A_{v}$ within the uncertainties of the radio data.

The $8 \mu \mathrm{m}$ emission from star-forming regions is generally attributed to young massive stars exciting aromatic hydrocarbon (PAH) emission bands or heating very small grains. Far ultraviolet emission from $B$ stars as well as $O$ stars can produce significant PAH emission via fluorescence (Peeters et al. 2004). Emission at $8 \mu \mathrm{m}$ depends on mechanisms involved in the formation and destruction of PAH molecules as well as the local SFR (Calzetti et al. 2005; Pérez-González et al. 2006; Dale et al. 2007).

From IRAC color-color plots, Elmegreen et al. (2006) find that the $8 \mu \mathrm{m}$ emission of most of the IRAC clumps in NGC 2207/IC 2163 is PAH dominated.

The mean value of the ratio $S_{\nu}(8 \mu \mathrm{m}) / S_{\nu}(6 \mathrm{~cm})$ for the fifteen prominent star-forming clumps in NGC 2207 is 18 with standard deviation $\sigma$ of the sample $=8$. Within the uncertainties, this mean value is the same as that for the giant radio H II regions in M81. We excluded the clump rc1 since it is probably either a radio supernova or a background quasar (see discussion below and in Section 6 ). In contrast, the mean value of this ratio for the ten prominent clumps on the IC 2163 eyelids is 34 with $\sigma=$ 16. The clumps IR 5, IR 8, IR 10 on the eyelids and IR 6 on the inner spiral arm of IC 2163 have values of $S_{\nu}(8$ $\mu \mathrm{m}) / S_{\nu}(6 \mathrm{~cm}) \geq 40$. The clumps on the eyelids are more luminous at $8 \mu \mathrm{m}$ but have the same mean $S_{\nu}(6 \mathrm{~cm})$ as the clumps in NGC 2207 (aside from feature $i$ ). As the ten eyelid clumps are detected in $\mathrm{H} \alpha$, they contain $O B$ associations, but they may in addition contain somewhat older star clusters with ages up to 100 million years and thus could be dominated at $8 \mu \mathrm{m}$ by $\mathrm{PAH}$ excitation involving $B$ stars rather than $O$ stars. Section 5 mentions another possible explanation for the high values of this ratio on the eyelids.

Although the source aperture used in Table 3 to measure the clumps is much greater in linear diameter $(1.2$ kpc vs $300 \mathrm{pc}$ ) than for the measurements of the M81 giant H II regions and thus may include emission, such as nonthermal radio emission from the spiral arm, unrelated to the $\mathrm{OB}$ associations, a number of clumps in NGC $2207 /$ IC 2163 have a value of the $8 \mu \mathrm{m}$ to $\lambda 6 \mathrm{~cm}$ flux density ratio similar to those of the M81 giant H II regions. Either subtraction of the local background has sufficiently removed the emission unrelated to the OB associations, or these are examples at $8 \mu \mathrm{m}$ analogous to the usual FIR-radio continuum correlation for galaxies (see Section 5.3)

The three brightest discrete $\lambda 6 \mathrm{~cm}$ sources in NGC $2207 /$ IC 2163 are the clumps IR 20 (which is feature $i$ ), rc1, and IR 12 in massive H I cloud N6. All three have low values of the $8 \mu \mathrm{m}$ to $\lambda 6 \mathrm{~cm}$ flux density ratio compared to the M81 giant H II regions. Nonthermal radio emission is significant in these three sources. Feature $i$ is the most luminous radio continuum source and contains the most luminous $\mathrm{H} \alpha$ region in the galaxy pair. VLA snapshots at $\lambda 6 \mathrm{~cm}$ and $\lambda 20 \mathrm{~cm}$ by Vila et al. (1990) indicate that feature $i$ is dominated by nonthermal radio emission, with a radio spectral index $\alpha$ (where $S_{\nu} \propto \nu^{-\alpha}$ ) ranging from 0.7 in its $1^{\prime \prime}$ radio core to 0.9 averaged over a $7.5^{\prime \prime} \times 7.5^{\prime \prime}$ box. Clump rc1, the second brightest discrete radio continuum source in Figure1, is unresolved in that image. Clump rc1 is reasonably bright in $U V M 2$ but does not appear as a significant clump in $\mathrm{H} \alpha$. The faintness in $\mathrm{H} \alpha$ is not the result of extinction, since rc1 is also rather faint at $8 \mu \mathrm{m}$. Thus $\mathrm{rc} 1$ is a nonthermal radio source. It may be a supernova remnant, a radio supernova, or a background quasar. Because we detect rc1 as an X-ray source, we defer further discussion of it to Section 6. Clump IR 12 lies in the region of brightest nonthermal radio emission on the $N E$ radio ridge.

Table 3 also lists the flux density ratio $S_{\nu}(8$ $\mu \mathrm{m}) / S_{\nu}(U V M 2)$, which is sensitive to the amount of extinction, to the distribution of extinction, to age, and to star-formation history. For the star-forming clumps that are bright in the ultraviolet, low values of this ratio indicate relatively low extinction. Very high values of this ratio may indicate lots of dust and considerable absorption. From the values of the $S_{\nu}(8 \mu \mathrm{m}) / S_{\nu}(U V M 2)$ ratio, it appears that clumps IR 21 , IR 26, u1, IR 138, and rc1 have relatively low extinction (e.g., values of $A_{v}$ similar to the M81 H II regions in Table 4), and that most of the clumps on the eyelids and feature $i$ suffer high extinction. 
The four brightest clumps in $U V M 2$ are $\mathrm{u} 1$, IR 21 , IR 19 , and IR 26. Relative to the $\mathrm{H} \alpha$ and radio continuum emission, the ultraviolet emission from u1 is displaced toward the outer edge of the arm. IR 138 is a bright $U V M 2$ clump that is faint in $\mathrm{H} \alpha$ and $8 \mu \mathrm{m}$ emission and not detected at $\lambda 6 \mathrm{~cm}$. On the $H S T$ image, it coincides with a short, thin string of sources in the NW interarm of NGC 2207. Clumps u1 and IR 138 are probably slightly older star complexes with little dust and with ultraviolet emission mainly from $B$ stars rather than $O$ stars. SN $1999 \mathrm{ec}$ at RA, DEC $(2000)=061616.18,-212210.1$ (Van Dyk et al. 2003) is $1.2^{\prime \prime} \mathrm{E}, 0.3^{\prime \prime} \mathrm{S}$ of the center of IR 21. IR 21 could be bright in the ultraviolet due to shock excitation of circumstellar gas by SN 1999ec (see comments about the corresponding X-ray source in Section 8). IR 21 resembles IR 19 and IR 26 in being fairly bright in $\mathrm{H} \alpha$, particularly prominent in $U V M 2$, and in having values for the $8 \mu \mathrm{m}$ to $\lambda 6 \mathrm{~cm}$ flux density ratio in the same range as the M81 giant radio $\mathrm{H}$ II regions. A simple interpretation is that these three clumps are OB associations with less extinction than most of the other clumps and that the $\mathrm{H} \alpha$, and most of the $\lambda 6 \mathrm{~cm}$ emission from IR 21 is from the $\mathrm{H}$ II region, not from the Type Ib SN 1999ec.

For eleven of the clumps, the value of the $8 \mu \mathrm{m}$ to $U V M 2$ flux density ratio is greater than or equal to 120. These include feature $i$, eight of the ten clumps on the eyelids, and the one clump on the inner spiral of IC 2163. It is not surprising to find that feature $i$ and the eyelid clumps suffer high extinction. HST observations (Elmegreen et al. 2006) reveal a large opaque dust cloud occulting part of feature $i$. The eyelids contain a large concentration of gas; this is in addition to the extinction from the outer arm of NGC 2207 cutting in front.

\section{LARGE-SCALE SHOCK FRONTS}

We consider the two large-scale shock fronts in this system, i.e., the eyelids of the eye-shaped oval in IC 2163 and the NE radio ridge in NGC 2207. Figure 5 shows the difference between the two shock fronts. The top panel in this figure displays the ratio of the $8 \mu \mathrm{m}$ surface brightness $I_{\nu}(8 \mu \mathrm{m})$ to the $\lambda 6 \mathrm{~cm}$ radio continuum surface brightness $I_{\nu}(6 \mathrm{~cm})$, and the bottom panel, the ratio of the $24 \mu \mathrm{m}$ surface brightness $I_{\nu}(24 \mu \mathrm{m})$ to $\lambda 6$ $\mathrm{cm}$ radio continuum surface brightness. To match the resolution of the $24 \mu \mathrm{m}$ image, we made a $\lambda 6 \mathrm{~cm}$ radio continuum image with a synthesized beam of $6^{\prime \prime}$ from the $\lambda 6 \mathrm{~cm}$ radio observations described in Section 2.2.

Two pixels in feature $i$ were hard saturated and thus blanked in the $24 \mu \mathrm{m}$ Basic Calibrated Data. For these two pixels, we substituted twice the $24 \mu \mathrm{m}$ surface brightness at the FWHM of the point-spread function.The donut-shaped appearance of feature $i$ and its environs in the $I_{\nu}(24 \mu \mathrm{m}) / I_{\nu}(6 \mathrm{~cm})$ ratio image (lower panel of Figure 5) results because the radio images have been cleaned of sidelobes (the diffraction patterns), but the Spitzer images have not.

The differences between the two large-scale shock fronts are also apparent from the comparison in Figure6 between the Spitzer $24 \mu \mathrm{m}$ and $70 \mu \mathrm{m}$ emission and the radio continuum emission at $\lambda 20 \mathrm{~cm}$ and $\lambda 3.5 \mathrm{~cm}$. The eyelids are brighter than the $N E$ radio ridge at $24 \mu \mathrm{m}$ and $70 \mu \mathrm{m}$ whereas the $N E$ radio ridge is appreciably brighter than the eyelids at $\lambda 20 \mathrm{~cm}$. The value of the ra- tio of FIR to $\lambda 20 \mathrm{~cm}$ emission varies with location in the galaxy pair. The radio continuum emission from NGC 2207 /IC 2163 is strongly nonthermal with a spectral in$\operatorname{dex} \alpha=0.92$ (where $S_{\nu} \propto \nu^{-\alpha}$ ) (Condon 1983), and thus nonthermal radio emission makes a smaller contribution at $\lambda 3.5 \mathrm{~cm}$ than at $\lambda 20 \mathrm{~cm}$. At $\lambda 3.5 \mathrm{~cm}$, the $N E$ radio ridge is a bit brighter than the eyelids.

\subsection{The Eyelids}

The eyelids are particularly bright in the $8 \mu \mathrm{m}$ and 24 $\mu \mathrm{m}$ images (Elmegreen et al. 2006) and also prominent in $\mathrm{H} \alpha$ and the radio continuum (see Figure 3 and Figure1, resp.). Aside from feature $i$, the brightest $70 \mu \mathrm{m}$ and $24 \mu \mathrm{m}$ emission in this galaxy pair is from the eyelids (see Figure6), and thus cooler dust and warmer dust are both strongly concentrated on the eyelids. The eyelid shock front has produced strongly enhanced star formation in a dusty environment. Emission from warm, very small grains at $24 \mu \mathrm{m}$ is considered a better tracer than $8 \mu \mathrm{m}$ emission of the SFR (Pérez-González et al. 2006; Wu et al. 2005; Calzetti et al. 2005; Dale et al. 2007). From Figure5, one sees that the values of the ratio $I_{\nu}(8 \mu \mathrm{m}) / I_{\nu}(6 \mathrm{~cm})$ on the eyelids are large compared to those of the M81 H II regions and large compared to typical values of prominent clumps on the spiral arms of NGC 2207. Similarly, along the northern eyelid the values of the ratio $I_{\nu}(24 \mu \mathrm{m}) / I_{\nu}(6 \mathrm{~cm})$ are greater than typical of the spiral arms of NGC 2207, but the effect at both eyelids is more significant at $8 \mu \mathrm{m}$ than at $24 \mu \mathrm{m}$.

The bright $8 \mu \mathrm{m}$ emission from the eyelids is almost entirely diffuse emission, not stellar photospheric emission. We checked this by using Pahre's method (Pahre et al. 2004) to remove the stellar contribution by scaling the surface brightness of the IRAC $1(3.6 \mu \mathrm{m})$ image.

Adolf Witt (private communication) suggests that increased collisions between dust grains due to the shock compression would cause more fragmentation of large grains and increase the number of small grains in the size range appropriate for emitting at $8 \mu \mathrm{m}$ and $24 \mu \mathrm{m}$. Thus collisional shattering of dust grains in the eyelid shock may account for the enhanced $8 \mu \mathrm{m}$ and $24 \mu \mathrm{m}$ emission from the eyelids by producing a greater number of very small grains with sizes appropriate for emitting at $8 \mu \mathrm{m}$ and $24 \mu \mathrm{m}$ and by forming more PAH molecules with sizes $\leq 10^{3} \mathrm{C}$ atoms close to the OB stars. According to Draine et al. (2007), it is the PAH particles with sizes $\leq 10^{3} \mathrm{C}$ atoms that produce most of the emission in the $7.7 \mu \mathrm{m}$ and $8.6 \mu \mathrm{m}$ bands.

\subsection{NE Radio Ridge}

Aside from feature $i$, the brightest large-scale radio continuum emission in this galaxy pair comes from the $N E$ radio ridge. In $\mathrm{H} \alpha, U V M 2,8 \mu \mathrm{m}$, or $24 \mu \mathrm{m}$ emission the $N E$ radio ridge is no brighter than other spiral arms of NGC 2207. Figure 5] shows that the values of the ratio of $8 \mu \mathrm{m}$ to $\lambda 6 \mathrm{~cm}$ surface brightness and, particularly, the ratio of $24 \mu \mathrm{m}$ to $\lambda 6 \mathrm{~cm}$ surface brightness are low on the $N E$ radio ridge compared to those of the M81 $\mathrm{H}$ II regions. The $\lambda 6 \mathrm{~cm}$ radio continuum emission is enhanced here without a commensurate effect on star formation. The magnetic field $B$ is compressed, increasing the synchrotron radio emission, but some condition for enhanced star formation is not fulfilled. In IC 2163, as 
well as NGC 2207, the companion side is brighter than the anticompanion side in the $\lambda 6 \mathrm{~cm}$ radio continuum. This is evidence of disk or halo scraping between the two galaxies.

The lower panel in Figure 1 compares the $\lambda 6 \mathrm{~cm}$ radio continuum image with the HST B-band image. South of $\mathrm{H}$ I massive cloud $\mathrm{N} 6$, the $N E$ radio ridge includes two spiral arms of NGC 2207 visible in the $B$-band image, one of which is backlit by IC 2163, and some emission from IC 2163. North of cloud N6, the inside edge of the $N E$ radio ridge coincides with an optical spiral arm, but the $\lambda 6 \mathrm{~cm}$ radio emission spreads significantly beyond the outer edge of this arm into the interarm and is brighter in the interarm.

A question is whether the $N E$ radio ridge is located in the thin disk of NGC 2207 or in a thick disk or in the halo on the back side of NGC 2207 (relative to us) between the two galaxies.

If the NE radio ridge lies in the disk of NGC 2207, it makes sense to discuss the ridge contrast (defined as the ridge-to-interarm radio disk at the inside edge of the ridge). At the $2.5^{\prime \prime}(425 \mathrm{pc})$ resolution of our high resolution $\lambda 6 \mathrm{~cm}$ radio continuum image, the shock width is somewhat resolved; going to higher resolution would probably not increase the ridge contrast by a significant factor. Along the inside edge of the NE radio ridge, the interarm radio disk is detected at a level of about $2 \times$ the rms noise in this image. Along much of this radio ridge from $\mathrm{PA}=102^{\circ}$ clockwise to $32^{\circ}$, the ridge contrast in surface brightness is greater than 4 and reaches a maximum of 10 at $\mathrm{H}$ I massive cloud N6. In the case of a strong non-cooling shock transverse to the magnetic field, the shock would increase the magnetic field $B$ by a factor of 4 . If equipartition or minimum energy or pressure equilibrium of cosmic-ray electrons and magnetic $B$ field applies (see, for example, Beck et al. 1985), then the intensity of radio synchrotron emission $I_{\nu} \propto B^{3+\alpha(n t)}$, where $\alpha(n t)$ is the nonthermal radio spectral index. The galaxy pair has $\alpha=0.92$. If the free-free component of the $\lambda 20 \mathrm{~cm}$ radio continuum emission is $\leq 10 \%$, then $\alpha(n t)=0.92$ to 1.1 . With $\alpha(n t)$ in this range and $I_{\nu} \propto B^{3+\alpha(n t)}$, we have the following. (a) A ridge contrast in surface brightness of 4 to 10 corresponds to a factor of 1.4 to 1.8 increase in the magnetic field. (b) A strong magnetohydrodynamic (MHD) shock, which increases $B$ by a factor of 4 , would increase $I_{\nu}$ by a factor of 200 to 300 . The latter is ruled out by the observed ridge contrast if the $N E$ radio ridge is in the disk. In the limiting case where the shock increases only the magnetic field and not the cosmic-ray electron density, $I_{\nu} \propto B^{1+\alpha(n t)}$ and a strong MHD shock, which increases $B$ by a factor of 4 , would increase $I_{\nu}$ by a factor of 14 to 18 for the above range of $\alpha(n t)$. This is somewhat greater than the observed ridge contrast of 4 to 10 . This limiting case is more likely to apply in the halo than in the thin disk. We conclude that if the $N E$ radio ridge is in the disk, it is a broad ridge of somewhat compressed magnetic field, not a strong MHD shock.

However, if the NE radio ridge is high off the midplane, then taking the measured ratio of the radio surface brightness on the $N E$ radio ridge to that of the interarm thin disk at its inside edge does not make sense, and thus a strong MHD shock may be allowed. The follow- ing comparison between the distributions of $\lambda 6 \mathrm{~cm}$ radio continuum emission, neutral gas, and cool dust in this system provides information relevant to the question of whether the NE radio ridge is in the disk. In NGC 2207, the distribution of $70 \mu \mathrm{m}$ emission (see Figure 6) and the distributions of $160 \mu \mathrm{m}$ emission (Elmegreen et al. 2006) and ${ }^{12} \mathrm{CO} J=1 \rightarrow 0$ emission measured at SEST (Thomasson 2004) generally correspond with the spiral arms. This indicates that molecular gas and the cool dust are cool fluids in the thin disk. The H I observations by Elmegreen et al. (1995a) of this galaxy pair (see also Figure 2) find that along the northern side of NGC 2207, the $\mathrm{H}$ I ridge consistently coincides with the spiral arm, but on the eastern and western sides of NGC 2207, the H I ridge line often lies in the interarm region, the massive $\mathrm{H}$ I clouds are usually in the interarm, and the $\mathrm{H}$ I gas has high velocity dispersion. The high velocity dispersion leads Kaufman et al. (1997) and Elmegreen et al. (2000) to suggest that the H I gas disk may be a few times thicker than normal. The H I disk may be flared on the companion and anti-companion sides of NGC 2207 to form a thick disk. In Figure 1 we see that the bright $\lambda 6 \mathrm{~cm}$ radio continuum emission from NGC 2207 generally coincides well with the stellar arms except on the $N E$ radio ridge. The most luminous radio continuum source on the $N E$ radio ridge is in the massive $\mathrm{H}$ I cloud $\mathrm{N} 6$; cloud $\mathrm{N} 6$ and this radio continuum source may be in the thick disk. Along the $N E$ radio ridge from cloud $\mathrm{N} 6$ clockwise to position angle $\mathrm{PA}=30^{\circ}$, the brightest $\lambda 6 \mathrm{~cm}$ radio continuum emission is in the interarm. The $\mathrm{H} \mathrm{I}$ emission here is also bright in the interarm. The $\lambda 6 \mathrm{~cm}$ emission and the $\mathrm{H}$ I emission on the $N E$ radio ridge appear clumpy. However, except for cloud N6, the clumps are not in one-to-one correspondence. More importantly, unlike the radio continuum, the $\mathrm{H}$ I emission from the $N E$ radio ridge is no brighter than from the opposite side of NGC 2207. This may be understood if a substantial fraction of the radio continuum emission in the $N E$ radio ridge originates in the halo.

On scales greater than about $2 \mathrm{kpc}$ in normal spiral galaxies, Adler et al. (1991) find that the ratio of ${ }^{12} \mathrm{CO}$ $J=1 \rightarrow 0$ intensity to radio continuum surface brightness is fairly constant. In NGC 2207, the $70 \mu \mathrm{m}, 160 \mu \mathrm{m}$, and ${ }^{12} \mathrm{CO} J=1 \rightarrow 0$ emission tend to be brighter on the eastern side of NGC 2207 than on its western side (aside from feature $i$ ). However, the difference between the two sides of NGC 2207 is less pronounced for the molecular gas and cool dust than for the radio continuum. Sensitive ${ }^{12} \mathrm{CO} J=1 \rightarrow 0$ mapping with higher spatial resolution than SEST would be useful here.

On the $N E$ radio ridge, we may be seeing a combination in which the bright radio continuum emission at the spiral arm is from the thin disk, some of the bright radio emission is from compressed magnetic fields in a thick disk (e.g., cloud N6), but most of the interarm radio emission is from the halo on the back side of NGC 2207 (relative to us) between the two galaxies. If the compressed magnetic field is in the halo and there is little neutral gas in the halo, then it is easy to understand why the $N E$ radio ridge is not a site of extended vigorous star formation. If the compressed magnetic field is in the thin disk, it seems necessary to invoke a time delay between compression of the magnetic field and compression 
of the neutral gas, which then leads to active star formation. We note that at the spiral arm, compression due to disk-scraping would add to the already existing compression of the spiral density wave to produce brighter radio continuum emission.

The lack of enhanced star formation on the $N E$ radio ridge is analogous to the lack of active star formation in most of the massive H I clouds in this galaxy pair. Much of the $\mathrm{H} \mathrm{I}$ in the thick disk may be at too low a volume density and thus there is a delay before molecular clouds form.

\subsection{Comments about IR to radio continuum ratios}

From Appleton et al. (2004) we adopt the notation $q_{\mathrm{IR}}$ $=\log \left(S_{\mathrm{IR}} / S_{1.4 \mathrm{GHz}}\right)$, where $S_{\mathrm{IR}}$ is the flux density in the Spitzer $8 \mu \mathrm{m}, 24 \mu \mathrm{m}$, or $70 \mu \mathrm{m}$ bands or in the IRAS FIR band, and $S_{1.4 \mathrm{GHz}}$ is the radio continuum flux density at $1.4 \mathrm{GHz}$. In Table 5] we compare the global values of $q_{\text {IR }}$ for NGC $2207 /$ IC 2163 with the median or mean values for galaxies in the Spitzer First-Look Survey from Appleton et al. (2004), for a sample of 30 or 35 starforming galaxies in the Spitzer First-Look Survey from Wu et al. (2005), for IRAS galaxies that do not contain a radio-loud AGN from Condon (1992), and for galaxies in the SINGS sample, where we used the data from Dale et al. (2007). For the SINGS sample, we omitted the galaxies with poor quality data that were excluded by Draine et al. (2007), and to have a more suitable comparison with NGC 2207/IC 2163, we also omitted the nine low metallicity galaxies listed by Draine et al. (2007). Including the E and S0 galaxies in the SINGS sample has little effect on the mean values of $q_{\mathrm{IR}}$ (see Table 5). For NGC $2207 /$ IC 2163, we used the $1.4 \mathrm{GHz}$ radio continuum flux density of $393 \pm 9 \mathrm{mJy}$ from the NRAO/VLA Sky Survey (NVSS) and the Spitzer $24 \mu \mathrm{m}$ and $70 \mu \mathrm{m}$ flux densities from Elmegreen et al. (2006) but revised the $8 \mu \mathrm{m}$ value to include the aperture correction for extended emission 9 and an improved global background subtraction. The values of $q_{\mathrm{FIR}}, q_{70}$, and $q_{8}$ for NGC 2207/IC 2163 are consistently below the medians or means of the above large samples of galaxies by 2.1 to $3.2 \sigma$, where $\sigma$ is the standard deviation of the sample. The value of $q_{24}$ for NGC $2207 /$ IC 2163 is $2 \sigma$ below the mean values of the samples in Wu et al. (2005) or SINGS, but only $1 \sigma$ below the sample in Appleton et al. (2004).

These values of $q_{\mathrm{IR}}$ are for entire galaxies. Table 5 also lists the mean values of $q_{24}$ and $q_{8}$ for the set of M81 H II regions in Table 4 obtained by using $S_{\nu}(20 \mathrm{~cm})$ values from Kaufman et al. (1987). The mean values of $q_{24}$ and $q_{8}$ for the M81 H II regions are consistent with the SINGS sample but, respectively, $2.2 \sigma$ and $1.8 \sigma$ greater than those of the sample of star-forming galaxies in $\mathrm{Wu}$ et al. (2005). The radio continuum from the M81 H II regions is optically-thin free-free emission, whereas the radio continuum of galaxies as a whole is dominated by nonthermal emission. If the galaxies have a radio spectral index $\alpha$ of 0.8 , then to obtain $\log \left[S_{\nu}(\mathrm{IR}) / S_{\nu}(4.86 \mathrm{GHz})\right]$, add $\alpha \log (4.86 \mathrm{GHz} / 1.4 \mathrm{GHz})$ to the $q_{\mathrm{IR}}$ values in Table 5 , i.e., add 0.43 to the mean $q_{\mathrm{IR}}$ values for the galaxy samples and 0.05 for the M81 H II regions. The resulting mean values for the $\mathrm{Wu}$ et al. (2005) sample are $S_{\nu}(8$ $\mu \mathrm{m}) / S_{\nu}(6 \mathrm{~cm})=22$ versus 19 for the M81 H II regions

\footnotetext{
9 from http://spider.ipac.caltech.edu/staff/jarrett/irac/
}

and $S_{\nu}(24 \mu \mathrm{m}) / S_{\nu}(6 \mathrm{~cm})=32$ versus 36 for the M81 H II regions. For the SINGS sample the resulting mean values are greater than these. Table 6 lists values of the flux densities at $\lambda 6 \mathrm{~cm}, 8 \mu \mathrm{m}$, and $24 \mu \mathrm{m}$ from the $N E$ radio ridge, from the box drawn around the eyelids in Figure1. and from the galaxy pair as a whole. The ratio of $8 \mu \mathrm{m}$ to $\lambda 6 \mathrm{~cm}$ radio continuum flux density equals 9 for $\mathrm{NGC}$ $2207 / \mathrm{IC} 2163$ as a whole and for the $N E$ radio ridge box. The ratio of $24 \mu \mathrm{m}$ to $\lambda 6 \mathrm{~cm}$ radio continuum flux density equals 15 for the galaxy pair as a whole and about 9 for the $N E$ radio ridge box (the latter value is uncertain because the box is only $14^{\prime \prime}$ wide). These values are low compared to those of the M81 H II regions and the mean values for the galaxies in the Wu et al. (2005) and SINGS samples. It is clear from Figure 5] and Table 6 that this is the result of excess $\lambda 6 \mathrm{~cm}$ radio continuum emission from large portions of NGC 2207, not just the $N E$ radio ridge box. The eyelid box contains the eyelids plus the region interior to the eyelids. The $8 \mu \mathrm{m}$ to $\lambda 6 \mathrm{~cm}$ flux density ratio $=19$ for the eyelid box is appreciably less than most of the values along the eyelids because (a) the box includes an outer arm of NGC 2207 which is bright at $\lambda 6 \mathrm{~cm}$ but faint at $8 \mu \mathrm{m}$, and (b) the outer part of the companion side of IC 2163 has enhanced radio continuum emission (produced by the same scraping as the $N E$ radio ridge).

The flux density ratio $S_{\nu}(24 \mu \mathrm{m}) / S_{\nu}(8 \mu \mathrm{m})$ depends on the strength of the PAH emission. It is sensitive to variations in PAH formation and destruction (Dale et al. 2007). Values of this ratio for NGC 2207/IC 2163 as a whole and for the eyelid box are listed in Table [6] and are consistent with those listed in Table 5 for the SINGS survey.

\section{X-RAY RESULTS}

The top panel in Figure 7 displays an X-ray image in the $0.5-10 \mathrm{keV}$ range obtained by combining data from the MOS camera with data from the pn camera and smoothing the image to get greater sensitivity. The same screening criteria as for the X-ray spectra (see Section 2 ) were applied. The bottom panel displays the $8 \mu \mathrm{m}$ Spitzer image with contours of X-ray emission from the MOS camera data only and with the source extraction boxes for the X-ray spectral analysis overlaid. The X-ray image used in the bottom panel has better spatial resolution (FWHM of the PSF $\sim 5^{\prime \prime}$ ) than the X-ray image in the top panel but lower sensitivity. For the discrete X-ray sources, we subtracted the local background by collecting background counts from a nearby region with the same area as the source box. With the MOS cameras, the entire field of interest fits onto a single CCD chip. With the pn camera, part of the southern eyelid of IC 2163 and parts of the discrete sources X2 and X7 in NGC 2207 fell in a gap between two CCDs

The X-ray images in Figure 7 clearly show the nucleus of NGC 2207 and nine other discrete X-ray sources labelled in the figure, as well as extended X-ray emission. No X-rays are detected at the position of the nucleus of IC 2163. There is little activity in the IC 2163 nucleus; it is faint (or very faint) in the radio continuum, ultraviolet, X-rays, $8 \mu \mathrm{m}$, and $24 \mu \mathrm{m}$ images. The nucleus of NGC 2207 is prominent in soft X-rays, hard X-rays, radio continuum, $\mathrm{H} \alpha, U V W 1$, and $8 \mu \mathrm{m}$ images, but rather faint in the $U V M 2$ image (as a result of extinction). The 
NGC 2207 nucleus is the only source in this galaxy pair that is bright in hard X-rays with $E>5 \mathrm{keV}$.

\subsection{The galaxy pair NGC 2207/IC2163}

The X-ray emission from the discrete X-ray sources and the extended emission, which may be from hot galactic gas, are mainly concentrated in NGC 2207. Aside from the NGC 2207 nucleus, most of the discrete X-ray sources lie on the spiral arms. Only a few of these correspond to the prominent IR or UV clumps discussed in Section 4.

The encounter models for this galaxy pair (Struck et al. 2005) predicted soft X-ray emission from diffuse hot plasma at the large-scale shock fronts. One goal of our XMM-Newton observations was to detect such emission. We find that neither the large-scale shock front along the eyelids nor the NE radio ridge appears enhanced in extended X-ray emission relative to the rest of this galaxy pair. X-ray absorption due to the large concentration of gas in the eyelids plus gas in the outer arm of NGC 2207 cutting in front of IC 2163 may explain why we do not detect significant, extended, soft X-ray emission from the eyelids. Part of the southern eyelid lies in the gap between two CCDs of the pn camera, but this is also where we find very high extinction in the ultraviolet for the star forming clumps (Section 4). Most of the $N E$ radio ridge has significantly less extinction than the eyelids. In the models of Struck et al. (2005), the NE radio ridge is attributed to disk or halo scraping. The lack of enhanced X-ray emission from the $N E$ radio ridge supports the suggestion that the bright radio continuum emission here arises in a region of significantly lower density than the thin disk, since no evidence that a shock due to disk or halo scraping has heated large quantities of gas to $\mathrm{X}$-ray temperatures.

The X-ray spectra of each individual source in Figure 7 plus the $N E$ radio ridge (denoted $\mathrm{RR}$ in Table 7 ) were fitted by an absorbed power law model with the absorption column density by the Milky Way fixed to the Galactic value given by Dickey \& Lockman (1990) and the absorption column density of the absorber at the location of NGC 2207 ( $\mathrm{z}=0.00941)$ left as a free parameter. The results of these fits are summarized in Table[7. The EPIC pn X-ray spectra of all of the sources except X2, $\mathrm{X} 5$, and $\mathrm{X} 7$ are displayed in Figure8. The MOS spectra of sources $\mathrm{X} 2$ and $\mathrm{X} 7$, which lie at the chip edge in the pn observations, are displayed in Figure 9. The pn and MOS spectra of X5 (the nucleus of NGC 2207) are displayed in Figure 10, Most of these X-ray spectra can be fitted with a power law with an energy spectral index of about $\alpha_{\mathrm{X}}=1.0$ (equivalent to a photon index $\Gamma$ of 2.0 ), except for X2 and X3, which show significantly steeper indices, and X5, which has a more complicated spectrum. The power-law model for X3 resulted in an unusually steep spectral index $\alpha_{X}=3.7_{-0.9}^{+2.6}$. A Raymond-Smith plasma is a better representation of the spectrum of $\mathrm{X} 3$, and this is what we list for X3 in Table 7.

If the 10 discrete sources in Table[7 are at the distance of IC 2163/NGC 2207 and emitting isotropically, then each has an X-ray luminosity $L_{x} \geq 2 \times 10^{39} \mathrm{erg} \mathrm{s}^{-1}$. Thus, each of the nine discrete sources in the disks of this galaxy pair is a ULX candidate. Since each could be a collection of sources, e.g., X-ray binaries, rather than a single object, Chandra high resolution observations and reobserving to look for variablility are necessary to check on this. From Chandra data on 32 nearby galaxies, Colbert et al. (2004) find that most of the discrete X-ray sources in the disks of spiral galaxies have spectra that fit an absorbed power-law with $\Gamma \approx 1-2$, appropriate for high-mass X-ray binaries associated with accretion-powered black holes, low-mass X-ray binaries, and ULXs and that only the Antennae merger pair (NGC 4038/39) contains more than three ULXs. From a study of ULXs in 82 galaxies, Swartz et al. (2004) conclude that $14 \%$ of ULX candidates in spiral galaxies are probably background sources. Only the following four galaxies in their sample have more than five ULXs: M82, M51, NGC 4038, and NGC 4486. From Chandra observations of the interacting starburst pair NGC 7714/7715, Smith et al. (2005) identify 11 candidate ULXs, only two of which are more luminous than the faintest discrete X-ray source listed in Table 7 for NGC 2207/IC 2163. If most of the candidates in NGC 2207 are ULXs, that would be a greater number than in a typical galaxy.

For comparison with the intrinsic absorption column densities $N_{\mathrm{H} \text {,intr }}$ obtained by fitting the X-ray continuum, Table7 lists the column density $N(\mathrm{HI})$ of the galaxy pair as measured in the 21-cm line VLA observations (Elmegreen et al. 1995a), averaged over the X-ray extraction aperture and not corrected for helium. In addition to $N(\mathrm{HI})$, there is a significant $\mathrm{H}_{2}$ column density in much of this galaxy pair (Thomasson 2004). The discrete sources, X2, X3, X8, X9 have no significant intrinsic absorption along the line of sight, which places them in the layers of the NGC 2207 gas disk closest to the observer. Source X1 may lie close to the midplane of the NGC 2207 gas disk. Most of the other discrete sources are more deeply embedded or located towards the farther side of the NGC 2207 gas disk.

We have the following identifications or possible identifications of the discrete X-ray sources (see Figure 4 for the labelling of the $8 \mu \mathrm{m}$ or ultraviolet clumps): $\mathrm{X} 5$ is the nucleus of NGC 2207, X10 is in the star-forming clump IR 11 on the eyelid of IC 2163 , X1 coincides with clump rc1, and X2 corresponds with clump IR 21, which contains SN 1999ec. The value of $N_{\mathrm{H} \text {,intr }}$ for source X10 is consistent with its location in clump IR 11 on the eyelid of IC 2163 behind an outer arm of NGC 2207; the H I column density of NGC 2207 at X10 is $0.32 \times 10^{22}$ atom

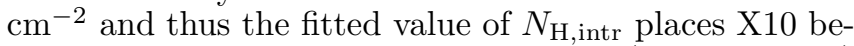
hind NGC 2207 but on the nearer side (relative to us) of the gas layer in the eyelids. The coincidence between X10 and star-forming clump IR 11 suggests that X10 is a high mass X-ray binary. Alternatively, Smith et al. (2005) note that some discrete X-ray sources in star forming regions may be due to SNRs with high-mass progenitors, rather than high mass X-ray binaries. Source X5 (the nucleus of NGC 2207) is the brightest X-ray source in the entire field. As a matter of fact it is the only $\mathrm{X}$-ray source in the hard X-ray band above $5 \mathrm{keV}$. We shall discuss sources X5 and X1 in Sections 6.2 and 6.3, respectively, and source X2 in Section 8.

The source denoted RR in Table 7 is not a discrete source but actually the entire $N E$ radio ridge, overlapping part of source X9. The X-ray spectrum of the $N E$ radio ridge suggests that the emission could be mainly 
from a collection of X-ray binaries. Thus the NE radio ridge is not unusual either in terms of its X-ray surface brightness or its X-ray spectrum. We do not detect SN $2003 \mathrm{H}$ on the $N E$ radio ridge as a discrete X-ray source.

\subsection{The nucleus of NGC 2207}

Figure 10 displays the pn and MOS spectra of the nucleus of NGC 2207 and shows that its emission contains a hard X-ray component. In order to increase the signal-tonoise ratio, all three spectra were fitted simultaneously in XSPEC. For all fits the absorption column density of the Milky Way was fixed to the Galactic value $\left(1.13 \times 10^{21}\right.$ $\mathrm{cm}^{-2}$; Dickey \& Lockman 1990). Fitting a single power law model to the data fails as the observed spectrum is double-humped; it yields a $\chi^{2} /$ dof $=125 / 65$ (where dof means degrees of freedom) and an X-ray spectral slope that appears to be unphysically flat with $\alpha_{\mathrm{X}}=-1.16$. The strong increase of the flux in hard X-rays suggests that a strong partial-covering absorber is present in the NGC 2207 nucleus. Models involving partial covering of an AGN X-ray source by dense blobs close to the central source have often been used to interpret the spectra of Narrow-Line Seyfert 1 galaxies (e.g., Grupe et al. 2007; Turner \& Miller 2009). We fitted a partial-covering absorber with a power law model to the data as shown in the upper left panel of Figure10. For this model, the fit is acceptable $\left(\chi^{2} /\right.$ dof $\left.=83 / 65\right)$ and yields for the partial covering absorber a column density of $N_{\mathrm{H}, \mathrm{pc}}=2.7 \times 10^{23}$ $\mathrm{cm}^{-2}$ and covering fraction of $f_{\mathrm{pc}}=0.95$ as listed in Table8. The lower left panel of Figure10 displays the contour plot between the column density and the covering fraction of the absorber. From this model we derived an unabsorbed flux in the observed $0.3-10.0 \mathrm{keV}$ band of $2.1 \times 10^{-13} \mathrm{ergs} \mathrm{s}^{-1} \mathrm{~cm}^{-2}$ which converts to a luminosity of $0.4 \times 10^{41} \mathrm{ergs} \mathrm{s}^{-1}$ in the $0.3-10.0 \mathrm{keV}$ band. This is the luminosity of a low-luminosity Seyfert galaxy, most-likely Seyfert 2 galaxy. There is excess emission at energies below $1 \mathrm{keV}$, which can be interpreted as strong emission lines from ionized gas. Such emission lines from ionized gas have been reported in several cases of partial covering absorption sources, such as the Narrow-Line Seyfert 1 Galaxies Mkn 1239 and Mkn 335 (Grupe et al. 2004, 2007, 2008) or the Seyfert 2 galaxy NGC 5643 (Guainazzi et al. 2004). Our data, however, do not allow us to constrain any parameters of this ionized gas.

The pn and MOS spectra can be fitted alternatively by an absorbed blackbody plus power law model with no intrinsic absorption. This fit is shown in the upper right panel of Figure 10 and listed in Table 8 . The lower right panel of Figure 10 displays the blackbody and the power law components separately. The blackbody temperature is $k T=163_{-19}^{+29} \mathrm{eV}$ and the energy spectral index $\alpha_{\mathrm{X}}=-$ $1.56_{-0.16}^{+0.14}$ with $\chi^{2} / \mathrm{dof}=74 / 62$. Still, the residuals below $1 \mathrm{keV}$ remain. Although the $\chi^{2} /$ dof of the blackbody plus power-law model suggests a slightly better fit compared with the partial-covering absorber model, an F-test shows this is only a slight improvement $(\mathrm{F}$ - value $=2.51$ and a probability of a null random result $P=0.067$ ) The energy spectral slope of the hard X-ray power law is unphysically flat, i.e., would require too many higher energy relativistic electrons compared to lower energy relativistic electrons. Therefore we conclude that the most likely model to explain the X-ray spectrum of the nucleus of
NGC 2207 is the power law model with partial-covering absorption.

The nucleus has an $\mathrm{H} \alpha$ flux of $1.58 \times 10^{-14} \mathrm{ergs} \mathrm{s}^{-1}$ $\mathrm{cm}^{-2}$ and a $\lambda 6 \mathrm{~cm}$ flux density of $0.37 \mathrm{mJy}$ (equivalent to a $\lambda 6 \mathrm{~cm}$ radio continuum luminosity of $5 \times 10^{19} \mathrm{~W} \mathrm{~Hz}^{-1}$ ). These values are similar to those of the eyelid clump IR 10. Half of the clumps in Table 3 have greater values of the $\lambda 6 \mathrm{~cm}$ flux density than the NGC 2207 nucleus, and thus the nucleus is relatively radio quiet for an AGN.

\subsection{Source $X 1$}

Figure11 provides a detailed view of X-ray source X1 with X-ray contours from the MOS data overlaid on the $H S T B$-band in the left panel and overlaid on the $U V M 2$ image in the right panel. The plus sign marks the location of the unresolved, nonthermal radio continuum clump rc1, and the width of the plus sign is the HPBW of the $\lambda 6 \mathrm{~cm}$ synthesized beam. The main X-ray emission is centered on a collection of blue star clusters, the most prominent of which lies close to the discrete radio source. With $A_{\mathrm{v}} / N_{\text {gas }}=0.53 \times 10^{-21} \mathrm{mag}$ per atom $\mathrm{cm}^{-2}$ from Bohlin et al. (1978) for solar neighborhood metallicity, the intrinsic X-ray absorption column density of $\mathrm{X} 1$ is equivalent to $A_{\mathrm{v}}=0.6_{-0.6}^{+1.1} \mathrm{mag}$. The value of the $8 \mu \mathrm{m}$ to $U V M 2$ flux density ratio (see Section 4) suggests that the clump rc1 suffers little extinction. Other evidence of low extinction is the close resemblance between the $B$ band and $U V M 2$ images. The $\mathrm{H}$ I line profiles obtained by Elmegreen et al. (1995a) show no evidence for an absorption feature at $\mathrm{rc} 1$, but the $\mathrm{H}$ I data has low spatial resolution of $13.5^{\prime \prime} \times 12^{\prime \prime}$.

Fitting a 2D Gaussian plus a flat baseline to the unresolved source rc1 on our $\lambda 6 \mathrm{~cm}$ radio continuum image with $2.5^{\prime \prime}$ resolution gives $S_{\nu}(6 \mathrm{~cm})=1.12 \pm 0.03 \mathrm{mJy}$. If the radio source rc1 is in NGC 2207 (rather than a background quasar) and is isotropic, it has a $\lambda 6 \mathrm{~cm}$ radio continuum luminosity $L_{\nu}(6 \mathrm{~cm})=1.6 \times 10^{20} \mathrm{~W} \mathrm{~Hz}^{-1}$, which is $230 \times L_{\nu}(6 \mathrm{~cm})$ of Cas A (taking $L_{\nu}(6 \mathrm{~cm})$ of Cas $\mathrm{A}$ as $7 \times 10^{17} \mathrm{~W} \mathrm{~Hz}^{-1}$ from Weiler et al. 1989). This $\lambda 6$ $\mathrm{cm}$ luminosity lies in the range of radio supernovae and in the range of the very brightest SNRs. Neff \& Ulvested (2000) find the three brightest, discrete, nonthermal radio sources in the Antennae merger pair have $L_{\nu}(6 \mathrm{~cm})$ in the range $8 \times 10^{19}$ to $2 \times 10^{20} \mathrm{~W} \mathrm{~Hz}^{-1}$ and attribute these to SNRs. Each is slightly extended in their high resolution radio continuum observations. The brightest of these Antennae SNRs is listed as an ULX candidate by Swartz et al. (2004) with a $L_{\mathrm{x}}$ in the $0.5-8 \mathrm{keV}$ band of $18 \pm 11 \times 10^{39} \mathrm{erg} \mathrm{s}^{-1}$ and $\Gamma=2.54$, i.e., similar in $\mathrm{X}$-ray spectrum and somewhat more luminous in X-rays than our source X1, which has $L_{\mathrm{x}}=7 \times 10^{39} \mathrm{erg} \mathrm{s}^{-1}$.

Possible interpretations of the source X1/rc1 are a radio supernova, a supernova remnant, a background quasar, or a chance superposition of a ULX in NGC 2207 with a background radio quasar. It is clear from Figure 6 that rc1 was brighter on 2003 Jan 14 when the $\lambda 3.5$ $\mathrm{cm}(8.46 \mathrm{GHz})$ radio continuum observations were taken than on 1990 October 11 when the line-free $\lambda 20 \mathrm{~cm}$ radio continuum observations were made: in the $\lambda 3.5 \mathrm{~cm}$ image, rc1 is much brighter than every other source in the galaxy pair except feature $i$, whereas no local surface brightness peak is seen at the location of rc1 in the $\lambda 20 \mathrm{~cm}$ radio continuum image. Also, no local surface 
brightness peak at rc1 is visible in the figure in Condon (1983) which displays a $\lambda 20 \mathrm{~cm}$ radio continuum image of NGC $2207 /$ IC 2163 with $7.6^{\prime \prime} \times 6^{\prime \prime}$ resolution. For a quantitative comparison, we measured the flux density of a $21^{\prime \prime} \times 21^{\prime \prime}$ box centered on $\mathrm{rc} 1$ in the above $\lambda 3.5$ $\mathrm{cm}$ image and in a $\lambda 6 \mathrm{~cm}$ image made from the uv-data used in Figure 11, i.e., B-configuration (high resolution) observations on 2001 April 14 plus D-configuration (low resolution) observations on 1995 May 13 . For the $21^{\prime \prime}$ box, $S_{\nu}(6 \mathrm{~cm})=3.38 \mathrm{mJy}$ and $S_{\nu}(3.5 \mathrm{~cm})=3.26 \mathrm{mJy}$. Part of this flux density is from emission unrelated to rc1. The $\lambda 6 \mathrm{~cm}$ flux density unrelated to rc1 in this box equals $3.38 \mathrm{mJy}-1.12 \mathrm{mJy}=2.26 \mathrm{mJy}$ ( where $S_{\nu}(6$ $\mathrm{cm})=1.12 \mathrm{mJy}$ for $\mathrm{rc}$, as measured on the high resolution image). If the spectral index $\alpha$ of the unrelated emission lies in the range 0.1 to 0.9 , then scaling the $2.26 \mathrm{mJy}$ from $\lambda 6 \mathrm{~cm}$ to $\lambda 3.5 \mathrm{~cm}$ gives $S_{\nu}(3.5 \mathrm{~cm})$ in the range 2.14 to $1.37 \mathrm{mJy}$ for the unrelated emission. Subtracting this from the measured $S_{\nu}(3.5 \mathrm{~cm})=3.26 \mathrm{mJy}$ of the $21^{\prime \prime}$ box in 2003 gives $S_{\nu}(3.5 \mathrm{~cm})$ for rc1 in 2003 in the range $1.12 \mathrm{mJy}$ to $1.69 \mathrm{mJy}$. As this is greater than or equal to the $\lambda 6 \mathrm{~cm}$ flux density of $\mathrm{rc} 1$ in the high resolution observations in 2001, either rc1 had increased in radio luminosity by 2003 and/or there is synchrotron self-absorption in the radio. Thus it is unlikely that rc1 is an SNR. The source rc1 could be a background quasar or a radio supernova. A young radio supernova whose spectrum is inverted because the emission at lower frequencies is still absorbed (Weiler \& Sramek 1988) is an example of a radio source with synchroton self-absorption that would be consistent with the observed properties of rc1.

The X-ray, radio, and ultraviolet emission from X1/rc1 may arise from parts of the source that differ in column density. For example Bregman \& Pildis (1992) find that the $\mathrm{X}$-ray and radio observations of the very luminous SN 1986J in NGC 891 are consistent with a model in which the radio emission is from the high temperature outward-moving shock whereas the soft X-ray emission is from the cooled material associated with the reverse shock.

\section{COMPARISON BETWEEN NGC 2207 AND NGC 2276}

We compare NGC 2207 with the spiral galaxy NGC 2276 since both galaxies are unusual in having a long ridge of bright radio emission from the outer part of the disk on one side of the galaxy. In NGC 2207 we call this feature the $N E$ radio ridge. The NGC 2276 radio continuum ridge is prominent in the images displayed in Hummel \& Beck (1995) and Condon (1983). Globally the NGC 2207/IC 2163 pair and NGC 2276 each have a radio continuum flux density that is 2.5 to 3 times higher than expected from the IRAS far-infrared flux.

NGC 2276 is a member of a small group of galaxies embedded in a large diffuse, intragroup, X-ray cloud. Unlike the NE radio ridge in NGC 2207, the large-scale bow-shock-like radio continuum ridge along the western edge of NGC 2276 is a site of active star formation and bright X-ray emission, visible in the Chandra observations by Rasmussen et al. (2006) and the ROSAT HighResolution Image by Davis et al. (1997). The stellar and gaseous disks in NGC 2276 truncate just beyond this shock-like feature whereas the $N E$ radio ridge in $\mathrm{NGC}$ 2207 is not the outermost spiral arm on the affected side of NGC 2207. The lopsided appearance of NGC 2276 has been attributed either to a tidal interaction with the ellipical galaxy NGC 2300 (Hummel \& Beck 1995; Davis et al. 1997) or to ram pressure from the hot intragroup gas (Rasmussen et al.|2006). From Chandra Xray observations, Rasmussen et al. (2006) find that NGC 2276 is moving supersonically at $850 \mathrm{~km} \mathrm{~s}^{-1}$ through the hot intragroup gas and that the ram pressure, which may have been acting for several $\times 10^{8} \mathrm{yr}$, could explain the observed compression of the $\mathrm{H}$ I gas and magnetic fields along the western edge of NGC 2276, leading to active star formation in this region. They argue that the X-ray emission from the western edge of NGC 2276 is dominated by hot plasma resulting from the vigorous star formation. This may be the reason why the $N E$ radio ridge in NGC 2207 is not bright in X-rays, since it is not a site of extended vigorous star formation. If the scraping between NGC 2207 and IC 2163 and the bright radio continuum emission from the $N E$ radio ridge are high off the disk of NGC 2207, this would explain why the observed $\mathrm{H}$ I column density contours are not unusually compressed on the $N E$ radio ridge and why there is no widespread active star formation there.

The IC 2163/NGC 2207 encounter models in Struck et al. (2005) predict a gas stream from IC 2163 impinging on NGC 2207, with shocks at a few hundred $\mathrm{km} \mathrm{s}^{-1}$ being pushed into both galaxies. However, the magnitude and direction of the tidal forces change as the encounter proceeds, and thus the compressed magnetic field in the $N E$ radio ridge may be a short-lived transient phenomenon.

\section{FEATURE $I$}

Feature $i$ is the most luminous radio continuum, $8 \mu \mathrm{m}$, $24 \mu \mathrm{m}$, and $\mathrm{H} \alpha$ source in the galaxy pair. Figure 12 displays the contours of emission from feature $i$ and environs in various wavebands overlaid on the $H S T B$-band image from observations made in 1996. As shown in this figure, feature $i$ is bright in $\lambda 6 \mathrm{~cm}$ radio continuum and $8 \mu \mathrm{m}$ emission but highly absorbed in the $U V M 2$ band. Comparison of the $U V M 2$ and $8 \mu \mathrm{m}$ images of this region provides an extinction map. Elmegreen et al. (2000) point out the opaque (optically thick even in $I$-band) conicallyshaped dust cloud (labelled here) with a bright compact cluster at its apex. The core of the radio source and the brightest $8 \mu \mathrm{m}$ and $24 \mu \mathrm{m}$ emission are centered on this cluster. The $U V M 2$ emission from the radio core and the conical dust cloud is highly absorbed and no X-ray emission is detected from either (see Figure 12).

In the radio continuum, feature $i$ is a core plus envelope source. Aside from a northern plume in the radio, it looks very similar in the $\lambda 6 \mathrm{~cm}$ and $8 \mu \mathrm{m}$ images: the extended emission fills a triangular region with E-W base $8^{\prime \prime}$ and N-S height $9.6^{\prime \prime}(=1.4 \mathrm{kpc} \times 1.7 \mathrm{kpc})$, which includes the cluster arcs of Elmegreen et al. (2000) and two superstar clusters identified by Elmegreen et al. (2001). Just north of the filled triangular region, the $\lambda 6 \mathrm{~cm}$ emission forms a plume with position angle $\mathrm{PA}=5^{\circ}$, whereas the $8 \mu \mathrm{m}$ emission is a little west of north, i.e., along the arm at $\mathrm{PA}=-15^{\circ}$. We take as the definition of feature $i$ the filled triangular region plus the radio plume. It has $S_{\nu}(6$ $\mathrm{cm})=4.67 \mathrm{mJy}$

We fit a simplified model consisting of the sum of two 2D Gaussians plus a flat baseline to the $\lambda 6 \mathrm{~cm}$ emis- 
sion from feature $i$ to represent the core plus envelope plus general arm emission. Table 9 lists the results obtained by using our $\lambda 6 \mathrm{~cm}$ image with the circular $2.5^{\prime \prime}$ synthesized beam and the results obtained by using our original $\lambda 6 \mathrm{~cm}$ image which has higher resolution in the $\mathrm{E}-\mathrm{W}$ direction $\left(\right.$ synthesized beam $=2.48^{\prime \prime} \times 1.30^{\prime \prime}, \mathrm{BPA}$ $=8^{\circ}$ ). These Gaussian models give for the core plus envelope $S_{\nu}(6 \mathrm{~cm})=4.6 \pm 0.07 \mathrm{mJy}$, attribute roughly $60 \%$ of the emission to the core, and find the core is slightly elongated along the same line as the opaque dust cloud (position angles $40^{\circ}$ for the core and about $40^{\circ}+180^{\circ}$ for the dust cloud). The opaque dust cloud has a projected length of $2^{\prime \prime}-3^{\prime \prime}$ (about twice the diameter of the radio core).

From VLA B configuration snapshot observations in 1986, Vila et al. (1990) measured $S_{\nu}(6 \mathrm{~cm})=1.4 \mathrm{mJy}$ for the $1^{\prime \prime}$ core and $3.4 \mathrm{mJy}$ for feature $i$ as a whole, whereas our combined VLA $\lambda 6 \mathrm{~cm}$ data from B configuration (high resolution) observations in 2001 and D configuration (low resolution) observations in 1995 give $2.66 \mathrm{mJy}$ for the $1^{\prime \prime}$ core and $4.66 \mathrm{mJy}$ for feature $i$ as a whole on our highest resolution image (see Table 9), i.e., $1.3 \mathrm{mJy}$ greater than the Vila et al. value for the core and $1.3 \mathrm{mJy}$ greater than the Vila et al. value for feature $i$ as a whole. Differences in flux density can arise from the missing short spacings and higher noise in the Vila et al. data and different ways of measuring the source. However since the difference in flux density between 1986 and 2001 is the same for the core and for feature $i$ as a whole and the $\lambda 6 \mathrm{~cm}$ flux density of the core in 2001 is nearly twice the Vila et al. value, we conclude that the $\lambda 6 \mathrm{~cm}$ flux density of the core increased by $1.3 \mathrm{mJy}$ between 1986 and 2001. This corresponds to an increase in $L_{\nu}(6 \mathrm{~cm})$ of $1.9 \times 10^{20} \mathrm{~W} \mathrm{~Hz}^{-1}$ if isotropic. As this lies in the luminosity range of radio supernovae, it seems likely that in 2001 a radio supernova was present in the core. From these data, we cannot determine the year when outburst in the core of feature $i$ occurred; Weiler \& Sramek (1988) point out examples of radio supernovae that remained bright in the radio for a number of years. A supernova in the core of feature $i$ may have been hidden from view optically by the high extinction. If our interpretation is correct, then NGC 2207 is remarkable in having had two optical supernovae (SN 1999ec and SN 2003H, both Type Ib) plus one radio supernova in recent years (i.e., between 1986 and 2003) and all with high-mass progenitors.

The simulation models by Elmegreen et al. (1995b) and Struck et al. (2005) for the NGC 2207/IC 2163 encounter estimate the inclination $i$ of the main disk of NGC 2207 as $25^{\circ}-35^{\circ}$ (relative to face-on) with the minor axis of the projection at $\mathrm{PA}=50^{\circ}-70^{\circ}$. The near side (relative to us) of NGC 2207 is the northeastern side. The opaque dust cloud is aligned nearly parallel to the minor axis of the projection of the main disk of NGC 2207 into the sky-plane. Elmegreen et al. (2000) point out a red $\mathrm{V}$-shaped structure with apex at the radio core and opening to the north (see Fig. 6 of that paper). The left fork of the $\mathrm{V}$ appears to be a continuation of the opaque dust cloud to the opposite side of the core, whereas the right fork of the $\mathrm{V}$ is aligned with the inside edge of the optical arm farther north, but straighter than most spiral-arm dust lanes, possibly as a result of extra compression of the original dust lane by the en- ergetic events in feature $i$. The radio plume is midway between the two forks of the $\mathrm{V}$. Given the orientation of the main disk of NGC 2207, the opaque dust cloud plus the left fork of the $\mathrm{V}$ could be outflow perpendicular to the plane of NGC 2207, generated at the radio core, i.e., the dark dust cone could be gas approaching us on the near side (relative to us) of the midplane and the left fork of the $\mathrm{V}$ could be gas receding from us on the far side and thus less prominent as an absorption feature because not obscuring light on the near side of the midplane.

Elmegreen et al. (2006) measure a $24 \mu \mathrm{m}$ flux density $S_{\nu}(24 \mu \mathrm{m})=248 \mathrm{mJy}$ for the $24^{\prime \prime} \times 24^{\prime \prime}$ field displayed in Figure[12. In the HiRES $24 \mu \mathrm{m}$ image in Velusamy et al. (2008) (which has a resolution of $1.9^{\prime \prime}$, a little better than that of the $8 \mu \mathrm{m}$ and $\lambda 6 \mathrm{~cm}$ images shown here), this flux density comes from a $7.5^{\prime \prime} \times 7.5^{\prime \prime}$ region centered on the radio core of feature $i$. This gives a $24 \mu \mathrm{m}$ to $\lambda 6 \mathrm{~cm}$ flux density ratio for feature $i$ of $248 \mathrm{mJy} / 4.67 \mathrm{mJy}=$ 53 , which is somewhat greater than the mean value $S_{\nu}(24$ $\mu \mathrm{m}) / S_{\nu}(6 \mathrm{~cm})=36 \pm 9$ for the M81 H II regions in Table 4 but similar to that of the most luminous giant radio $\mathrm{H}$ II region (K181) in M81. In Section 4 (see also Figure 5) we found that feature $i$ is underluminous at $8 \mu \mathrm{m}$ relative to its $\lambda 6 \mathrm{~cm}$ radio continuum emission when compared with the mean value for the M81 H II regions and with the mean value for clumps in NGC 2207 containing OB associations. For feature $i, S_{\nu}(24 \mu \mathrm{m}) / S_{\nu}(8 \mu \mathrm{m})=248$ $\mathrm{mJy} / 35 \mathrm{mJy}=7.1$, which is high compared to the mean value of 1.9 for the M81 H II regions in Table 4 and compared to the mean value for the SINGS sample (see Table 5), but similar to that of the dwarf (Im) starburst galaxy Mrk 33, which has $S_{\nu}(24 \mu \mathrm{m}) / S_{\nu}(8 \mu \mathrm{m})=6.6$ (Dale et al. 2007). Emission at $24 \mu \mathrm{m}$ is from warm very small grains whereas emission at $8 \mu \mathrm{m}$ is a combination of PAH emission bands and continuum emission from warm very small grains. The high value of the $24 \mu \mathrm{m}$ to $8 \mu \mathrm{m}$ flux density ratio of feature $i$ and the low value of the $8 \mu \mathrm{m}$ to $\lambda 6 \mathrm{~cm}$ flux density ratio suggest some $\mathrm{PAH}$ destruction by the radiation field of feature $i$ has depressed its $8 \mu \mathrm{m}$ emission.

In the HiRES $24 \mu \mathrm{m}$ image in Velusamy et al. (2008), feature $i$ appears as a filled elliptically-shaped region with minor axis/major axis ratio $=0.8$ and minor axis at $\mathrm{PA}$ $=50^{\circ}$. Given the orientation of the disk of NGC 2207, it is probably a filled circular region in the disk of NGC 2207.

A long-slit optical spectrum taken by Pierre Martin (private communication) with the CFHT MOS prior to 2000 cuts E-W through the core of feature $i$ and exhibits a normal H II region spectrum with no unusual line ratios. The long-slit optical spectrum of SN 1999ec (8" SSE of the radio core) taken by Matheson et al. (2001) has a PA of $-17^{\circ}$, a resolution of $6.3 \AA\left(380 \mathrm{~km} \mathrm{~s}^{-1}\right)$ FWHM at $\lambda 5000 \AA$, includes cluster arcs in feature $i$, and crosses the east fork of the red V. North of SN 1999ec, this spectrum shows normal H II region emission but the line profiles have a little asymmetry with a slightly more extended red wing, i.e., for the [O III] $\lambda 5007 \AA$ line, the center of a Gaussian fit is shifted systematically to the red relative to the intensity maximum with the shift increasing from $0.2 \pm 0.3 \AA$ at the southern cluster arcs to $0.6 \pm 0.3 \AA$ at the east fork of the red V. This may be an instrumental effect associated with non-centered sources projecting onto 
different positions on the chip. The optical emission-line velocities are generally consistent with the $\mathrm{H}$ I velocities given that the H I data from Elmegreen et al. (1995a) has low spatial resolution and a velocity dispersion of 56 $\mathrm{km} \mathrm{s}^{-1}$ at feature $i$. Neither of these optical spectra is suitable for looking for low velocity outflows from feature $i$. At the feature $i$ positions sampled by these two long slits, photoionization dominates, and these optical spectra show no components in feature $i$ at supernova or jet velocities.

Since photoionization dominates the optical spectra whereas the radio emission is nonthermal, we interpret feature $i$ as a mini-starburst and compare it with the central starburst in M82. The central $50^{\prime \prime} \times 15^{\prime \prime}$ starburst in M82 has $S_{\nu}(6 \mathrm{~cm})$ equal to $3.4 \pm 0.2 \mathrm{Jy}$ (Hargrave 1974), a radio spectral index $\alpha$ of 0.5 , and outflow over a wide range of solid angle (Seaquist \& Odegard 1991). If the M82 central starburst were at the $35 \mathrm{Mpc}$ distance of NGC 2207 instead of $3.6 \mathrm{Mpc}$, it would have a major axis of $5.1^{\prime \prime}$ (a little smaller than feature $i$ ) and $S_{\nu}(6 \mathrm{~cm})=36$ $\mathrm{mJy}$, which is 7.7 times the $\lambda 6 \mathrm{~cm}$ flux density of feature $i$ as a whole. Except in the $1^{\prime \prime}$ core of feature $i$, the $\lambda 6 \mathrm{~cm}$ radiation field in feature $i$ is significantly less intense than the average value for the M82 starburst. We estimate the value of $S_{\nu}(24 \mu \mathrm{m}) / S_{\nu}(6 \mathrm{~cm})$ of the central starburst in M82 for comparison with feature $i$. With a 25" aperture centered on the M82 starburst, Kleinman \& Low (1970) measured $S_{\nu}(22 \mu \mathrm{m})=120 \mathrm{Jy}$. Extrapolating to $24 \mu \mathrm{m}$ by taking $I_{\nu} \propto \nu^{-1}$ gives $S_{\nu}(24 \mu \mathrm{m})=130$ Jy for a $25^{\prime \prime}$ aperture. For the $50^{\prime \prime}$ extent of the radio emission from the M82 starburst, $S_{\nu}(24 \mu \mathrm{m})$ should be somewhat greater than $130 \mathrm{Jy}$, and thus $S_{\nu}(24 \mu \mathrm{m}) / S_{\nu}(6$ $\mathrm{cm}$ ) should be somewhat greater than $130 \mathrm{Jy} / 3.4 \mathrm{Jy}=$ 38. It appears that the ratio $S_{\nu}(24 \mu \mathrm{m}) / S_{\nu}(6 \mathrm{~cm})=53$ for feature $i$ is not unusual for a dusty starburst region.

The upper left panel in Figure12 displays contours of the X-ray emission from source X2. The core of feature $i$ is not detected as an X-ray source. The absence of soft X-rays could be the result of the high absorption seen from comparison of the $U V M 2$ and $8 \mu \mathrm{m}$ images. The $\mathrm{X}$-ray emission is generally south and SSE of feature $i$, and the brightest X-ray knot is centered $2.4^{\prime \prime}$ north of SN 1999ec. The upper right panel in this figure shows that the $U V M 2$ peak emission coincides with the supernova. As previously reported by Poolev (2007) from an archival search of Type Ib,c supernovae which used our XMM-Newton observations of NGC 2207, it seems likely that most of the X-ray emission from X2 is associated with the Type Ib supernova SN 1999ec. It is unusual for a Type Ib supernova to be seen as an X-ray source, and, interestingly, SN 1999ec is bright in X-rays 6 years after the optical SN was discovered. The X-ray emission may be from the supernova shock plowing into a circumstellar envelope. This makes it more plausible that the bright $U V M 2$ emission coinciding with SN 1999ec is due to shock excitation of circumstellar gas by the supernova. The X-ray spectral index of X2 is steeper than typical of an X-ray binary.

\section{CONCLUSIONS}

We presented the X-ray and UV data observed by $X M M-N e w t o n$ and new $\lambda 6 \mathrm{~cm}$ radio continuum observations of the interacting galaxies NGC 2207/IC 2163. When combined with our previous observations in $\mathrm{H} \alpha$,
HST B-band, Spitzer infrared, H I, and SEST ${ }^{12} \mathrm{CO}$ $J=1 \rightarrow 0$, these data allow us to see the effects of the grazing encounter in producing large scale shocks, to study star complexes, supernovae, and the galactic nuclei in this pair, and to identify possible ULX candidates.

In X-rays we detect the nucleus of NGC 2207, nine possible ULX candidates, and extended X-ray emission, mainly from NGC 2207. One of the discrete X-ray sources corresponds to SN 1999 ec and another has brightened in the radio in recent years and could be a radio supernova or a background quasar. The bright $U V M 2$ and X-ray emission from SN 1999ec may be from shock excitation of circumstellar gas by the supernova. The strongest source in our XMM-Newton X-ray observations of NGC 2207/ IC 2163 is the nucleus of NGC 2207. It is the only hard X-ray source in the field. The preferred model for its X-ray spectrum is a power law with a partial covering absorber. Most likely this is a strongly absorbed, low-luminosity, Seyfert 2 AGN. However, optical spectroscopy is needed to confirm this assumption.

We measured values of the ratio of $8 \mu \mathrm{m}$ to $\lambda 6 \mathrm{~cm}$ radio continuum flux density for the prominent, kpc-sized, star-forming clumps in the galaxy pair and compared them with those for the M81 H II regions whose radio continuum is dominated by optically-thin free-free emission. For the bright clumps in NGC 2207, the mean value of this ratio equals 18 , which is the same as for giant radio H II regions in M81, within the uncertainties. For the bright clumps on the rim (the eyelids) of the eye-shaped oval in IC 2163 , the mean value is nearly a factor of two greater.

There are two types of large-scale fronts in this galaxy pair: the eyelids of the eye-shaped oval in IC 2163 and the $N E$ radio ridge on the companion side of NGC 2207. The eyelid shock front is produced by radial inflow and convergence of orbits and has vigorous star formation in a dusty environment. In the eyelids the ratio of $8 \mu \mathrm{m}$ to $\lambda 6 \mathrm{~cm}$ surface brightness is appreciably greater than in the M81 giant H II regions. This could be the result of heating mainly by $B$ stars instead of $O$ stars and/or fragmentation of dust grains by collisions in the shock region and formation of more $\mathrm{PAH}$ molecules close to the $O B$ stars. The eyelids are located behind the outer part of NGC 2207; this may explain why they are not bright in extended soft X-ray emission.

The NE radio ridge in NGC 2207 is particularly bright in the radio continuum but not in any of the tracers of recent star formation. Values of the ratios of $8 \mu \mathrm{m}$ to $\lambda 6 \mathrm{~cm}$ surface brightness and $24 \mu \mathrm{m}$ to $\lambda 6 \mathrm{~cm}$ surface brightness are low on the $N E$ radio ridge compared to those of the M81 H II regions. Unlike the bright radio ridge in the outer part of NGC 2276, the $N E$ radio ridge is not enhanced in extended X-ray emission relative to the rest of galaxy; this is probably because it is not a site of active star formation. The NE radio ridge, which previous models attributed to disk or halo scraping, is simply due to compression of the magnetic field and may be mainly in the halo on the back side of NGC 2207 (between the two galaxies). If the bright radio continuum emission from the $N E$ radio ridge originates high off the disk of NGC 2207 in a region with little neutral gas, that would explain the lack of widespread vigorous star formation and the lack of unusually compressed H I column density contours on the $N E$ radio ridge. 
For NGC 2207/IC 2163, the global values of the ratios of infrared to radio continuum flux density in the Spitzer $8 \mu \mathrm{m}, 24 \mu \mathrm{m}$, and $70 \mu \mathrm{m}$ bands and the IRAS FIR are significantly below the medians/means for large samples of galaxies. This is the result of excess radio continuum emission from large portions of NGC 2207, not just the $N E$ radio ridge.

We find evidence that a radio supernova was present in the core of feature $i$ in 2001. If so, then NGC 2207 had two optical supernovae and one radio supernova in recent years. The $\lambda 6 \mathrm{~cm}$ radio continuum luminosity of feature $i$ on an outer arm of NGC 2207 is $13 \%$ of the central starburst in M82. In linear size, feature $i$ is a little larger than the M82 starburst. Like the M82 starburst, feature $i$ is a dusty starburst region with radio continuum emission that is mainly nonthermal and with a $24 \mu \mathrm{m}$ to $\lambda 6 \mathrm{~cm}$ flux density ratio somewhat greater than the mean value for the M81 giant H II regions. Whereas the M82 starburst has outflow perpendicular to the disk which is bright in $\mathrm{X}$-rays, our only indication of outflow perpendicular to the disk in feature $i$ is the peculiar morphology of the dust structures.

This work is based on observations obtained with $X M M-N e w t o n$, an ESA science mission with instruments and contributions directly funded by ESA Member States and NASA. We gratefully acknowledge support from NASA Goddard Grant NNG05GR10G to M.K., D.G., and D.M.E. This research has made use of the NASA/IPAC Extragalactic Database (NED) which is operated by the Jet Propulsion Laboratory, Caltech, under contract with the National Aeronautics and Space Administration. We also used a radio continuum image from the NRAO VLA public archives. We thank Tom Matheson and Perry Berlind for sending us FITS images of their long-slit spectrum of SN 1999ec. We thank Thangasamy Velusamy for sending us the HiRes FITS image of the Spitzer $24 \mu \mathrm{m}$ emission. We thank Pierre Martin for providing figures displaying his long-slit spectrum of the core of feature $i$ and his measurements of line ratios.

\section{REFERENCES}

Adler, D.S., Allen, R.J., \& Lo, K.Y. 1991,ApJ, 382, 475

Appleton, P.N., et al. 2004 ApJS, 154, 147

Appleton, P.N., et al. 2006, ApJL, 636, L51

Arnaud, K. A. 1996, ASP Conf. Ser. 101: Astronomical Data Analysis Software and Systems V, 101, 17

Beck, R., Klein, U., \& Krause, M. 1985, A\&A, 152, 137

Bohlin, R.C., Savage, B. D., \& Drake, J.F. 1978, ApJ, 224, 132

Bregman, J.N. \& Pildis, R.A. 1992, ApJL, 398, L107

Calzetti,D., et al. 2005, ApJ, 633, 871

Colbert, E.J.M, Heckman, T.M., Ptak, A.E., Strickland, D.K., \& Weaver, K.A.2004, ApJ, 602, 231

Condon, J.J. 1983, ApJS, 53,459

Condon, J.J. 1992, ARA\&A, 30, 575

Condon, J.J., Helou, G., Sanders, D.B., \& Soifer, B.T. 1993, AJ, 105,1730

Condon, J.J., Helou, G., \& Jarrett, T.H. 2002, AJ, 123, 1881

Dale, D.A. et al 2007, ApJ, 655, 863

Dame, T.M., Hartmann, D., \& Thaddeus, P. 2001, ApJ, 547, 792

Davis, D.S., Keel, W.C., Mulchaey, J.S., \& Henning, P.A. 1997, AJ, 114,613

Dickey, J.M., \& Lockman, F.J. 1990, ARA\&A, 28, 215

Draine, B.T., et al. 2007, ApJ, 663, 866

Elmegreen, B.G., Kaufman, M., \& Thomasson, M. 1993, ApJ, $412,90$.

Elmegreen, D.M., Kaufman, M., Brinks, E., Elmegreen, B.G., \& Sundin, M. 1995a, ApJ, 453, 100

Elmegreen, B.G., Sundin, M., Kaufman, M., Brinks, E., \& Elmegreen, D.M. 1995b, ApJ, 453, 139

Elmegreen, B.G., Elmegreen, D.M., Brinks, E., Yuan, C., Kaufman, M., Klaric, M., Montenegro, L, Struck, C., and Thomasson, M. 1998, ApJL, 503, L119

Elmegreen, B.G., et al. 2000, AJ, 120, 630

Elmegreen, D.M., Kaufman, M. Elmegreen, B.G., Brinks, E., Struck, C., Klaric, M., \& Thomasson, M.2001, AJ, 121, 182

Elmegreen, D.M., Elmegreen, B.G., Kaufman, M., Sheth, K., Struck, C., Thomasson, M., \& Brinks, E. 2006, ApJ, 642, 158

Griffith, M.R., Wright, A.E., Burke, B.F., \& Ekers, R.D. 1994 ApJS, 90, 179

Grupe, D., Mathur, S., \& Komossa, S. 2004b, AJ, 127, 3161

Grupe, D., Komossa, S., \& Gallo, L.C. 2007, ApJL, 668, L111

Grupe, D., Komossa, S., Gallo, L.C., Fabian, A.C., Larrson, J.,

Pradhan, A.K., Xu, D., \& Miniutti, G. 2008, ApJ, 681, 982

Guainazzi, M., Rodriguez-Pascual, P., Fabian, A.C., Iwasawa, K., \& Matt, G. 2004, MNRAS, 355, 297

Hargrave 1974, MNRAS, 168, 491

Hummel, E. \& Beck, R. 1995, A\&A, 303, 691

Jansen, F., et al. 2001, A\&A, 365, L1
Kaufman, M., Bash, F.N., Kennicutt, R.C., \& Hodge, P.W. 1987, ApJ, 319, 61

Kaufman, M., Brinks, E., Elmegreen, D.M., Thomasson, M.,

Elmegreen, B.G., Struck, C. \& Klaric, M. 1997, AJ, 114, 2323

Kleinman \& Low 1970, ApJL, 161, L203

Mason, K.O., et al. 2001, A\&A, 365, L36

Matheson, T.,Filippenko, A.V., Li, W., Leonard, D.C. 2001, ApJ, 121,1648

Matsumoto, H., et al. 2001, ApJL, 545, L25

Neff, S.G. \& Ulvestad, J.S. 2000, AJ, 120, 670

Pahre, M.A., Ashby, M.L.N., Fazio, G.G., \& Willner, S.P. 2004,

ApJS, 154, 235

Pérez-González, P.G. et al. 2006, ApJ, 648, 987

Peeters, E., Spoon, H.W.W., \& Tielens, A.G.G.M. 2004, ApJ, 613, 986

Pooley, D. 2007, in "Supernova 1987A: 20 Years After:

Supernovae and Gamma-Ray Bursters," AIP Conf. Proc., 937, p. 381 (eds. Stefan Immler, Kurt Weiler, Richard McCray)

Rasmussen, J., Ponman, T.J., \& Mulchaey, J.S. 2006, MNRAS, 370,453

Savage, B.D., \& Mathis, J.S. 1979, ARA\&A, 17, 73

Schlegel, D. J., Finkbeiner, D. P., \& Davis, M. 1998, ApJ, 500, 525

Seaquist, E.R.\& Odegard, N. 1991, ApJ, 369, 320

Smith, B.J., Struck, C., \& Nowak, M.A., 2005, AJ, 129, 1350

Struck, C., Kaufman, M., Brinks, E., Thomasson, M., Elmegreen,

B., \& Elmegreen, D. 2005, MNRAS, 364, 69

Strüder, L., et al. 2001, A\&A, 365, L18

Sulentic, J.W., Rosado, M., Dultzin-Hacyan, D.,

Verdes-Montenegro, L., Trinchiero, G., Xu, C., \& Pietsch, W. 2001, AJ, 122, 2993

Swartz, D.A., Ghosh, K.K., Tennant, A.F., \& Wu, K. 2004, ApJS, 154, 519

Tananbaum, H., Peters, G., Forman, W., Giacconi, R., Jones, C., \& Avni, Y. 1978, ApJ, 223, 74

Tananbaum, H., et al. 1979, ApJL, 234, L9

Thomasson, M. 2004, in "The Neutral ISM in Starburst Galaxies," ASP Conf. Ser., 320, p. 81 (eds. Susanne Aalto, Susanne Hüttemeister, Alan Pedlar)

Turner, M.J., et al. 2001, A\&A, 365, L27

Turner, M.J., \& Miller, L., 2009, A\&AR, 17, 47

Van Dyk, S.D., Li. W., \& Filippenko, A.V. 2003, PASP, 115, 1

Velusamy, T., Marsh, K.A., Beichman, C.A., Backus, C.R., \&

Thompson, T.J. 2008, AJ, 136, 197

Vila, M.B., Pedlar, A., Davies, R.D., Hummel, E., \& Axon, D.J. 1990, MNRAS, 242, 379

Voges, W., Aschenbach, B., Boller, T., et al. 1999, A\&A, 349, 389

Weiler, K.W. \& Sramek, R.A. 1988, ARA\&A, 26, 295 
Weiler, K.W., Panagia, N., Sramek, R.A., van der Hulst, J.M., Roberts, M.S., \& Nguyen, L. 1989, ApJ, 336, 421

Wu, H., Cao, C., Hao, C.N., Liu, F.S., Wang, J.L., Xia, X.Y., Deng, Z.G. \& Young, C.K.S. 2005, ApJL, 632, L79 

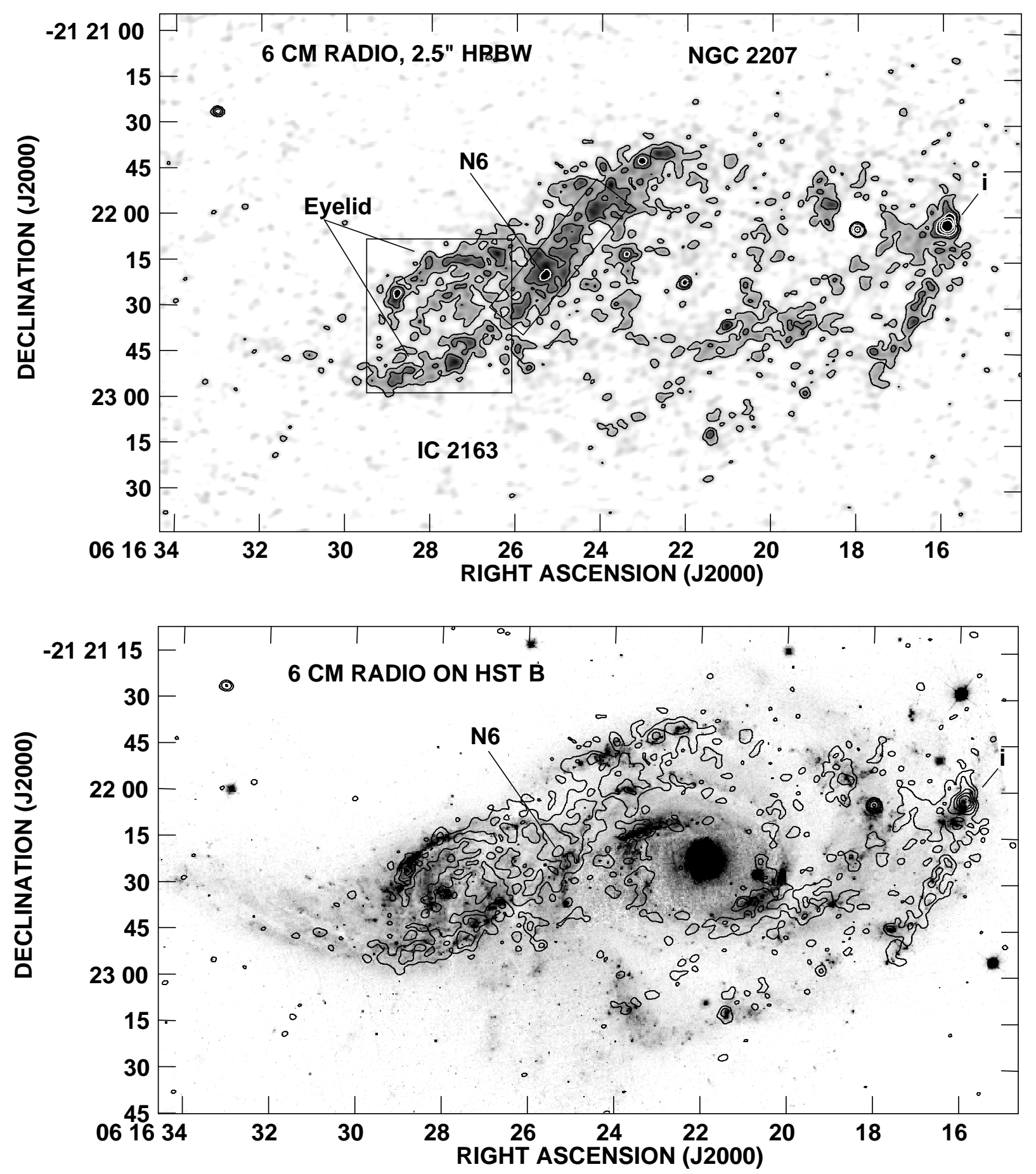

FIG. 1. - Top: Grayscale plus contour display of the $\lambda 6 \mathrm{~cm}$ radio continuum image. The rms noise is $0.016 \mathrm{mJy}^{\mathrm{beam}}{ }^{-1}$, equivalent to $T_{b}=0.13 \mathrm{~K}$, and the contours are at $4,8,16,32$, and 64 times the rms noise. The label $i$ marks feature $i$, the long tilted box marks the location of the $N E$ radio ridge, and the label N6, the location of massive H I cloud N6. Bottom: the same contours of $\lambda 6 \mathrm{~cm}$ emission overlaid on the $H S T B$-band image. 

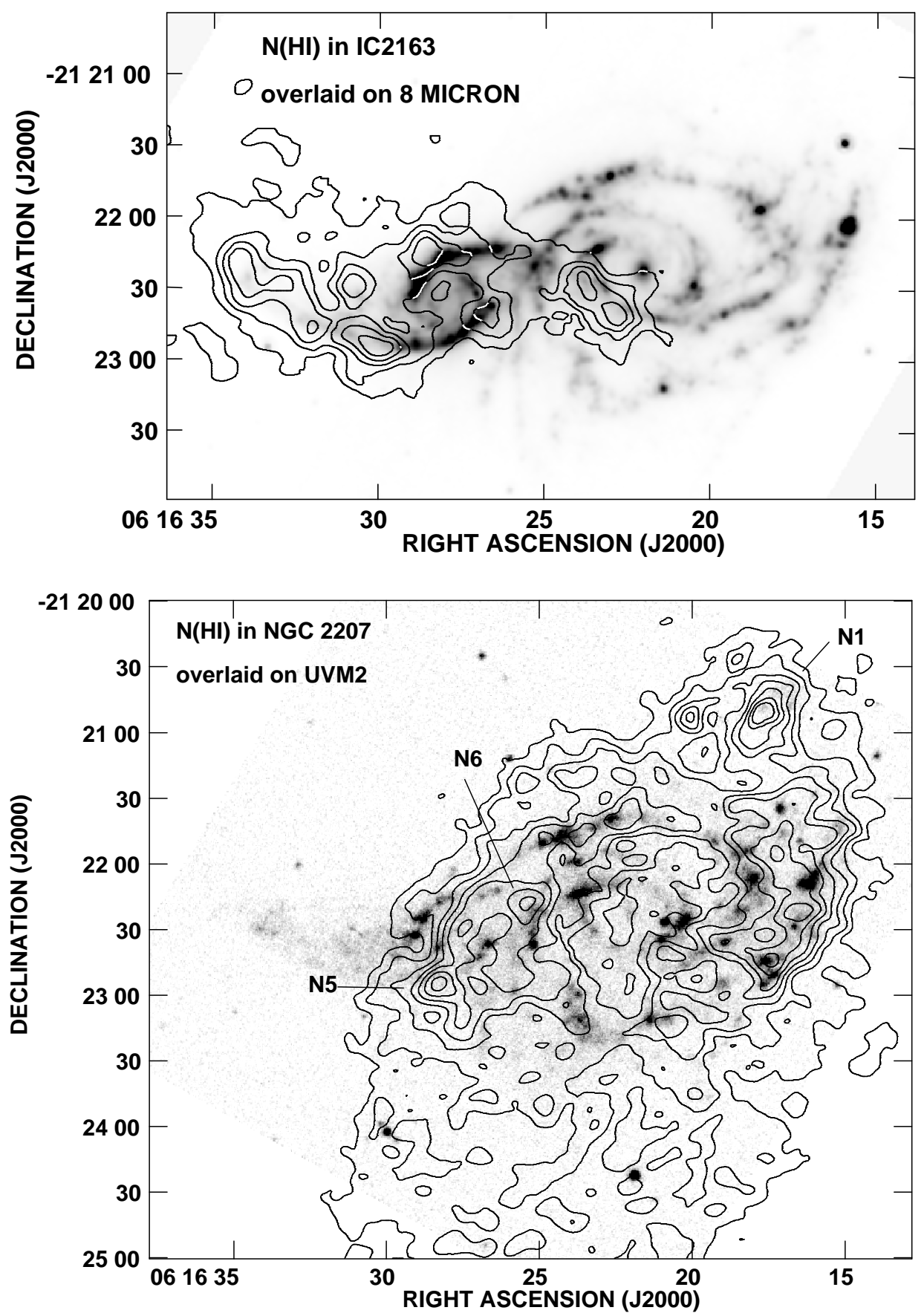

FIG. 2.- Top: $N(\mathrm{HI})$ contours of IC 2163 overlaid on the Spitzer $8 \mu \mathrm{m}$ image of NGC 2207/IC 2163. Bottom: $N($ HI) contours of NGC 2207 overlaid on the UVM2 image of the galaxy pair. Contour levels are $1.25 \times 10^{20}$ atoms cm ${ }^{-2}$ times $5,10,15,20,25,30$, and 35 . Massive H I clouds N1, N5, and N6 are three of the six massive H I clouds in NGC 2207. 

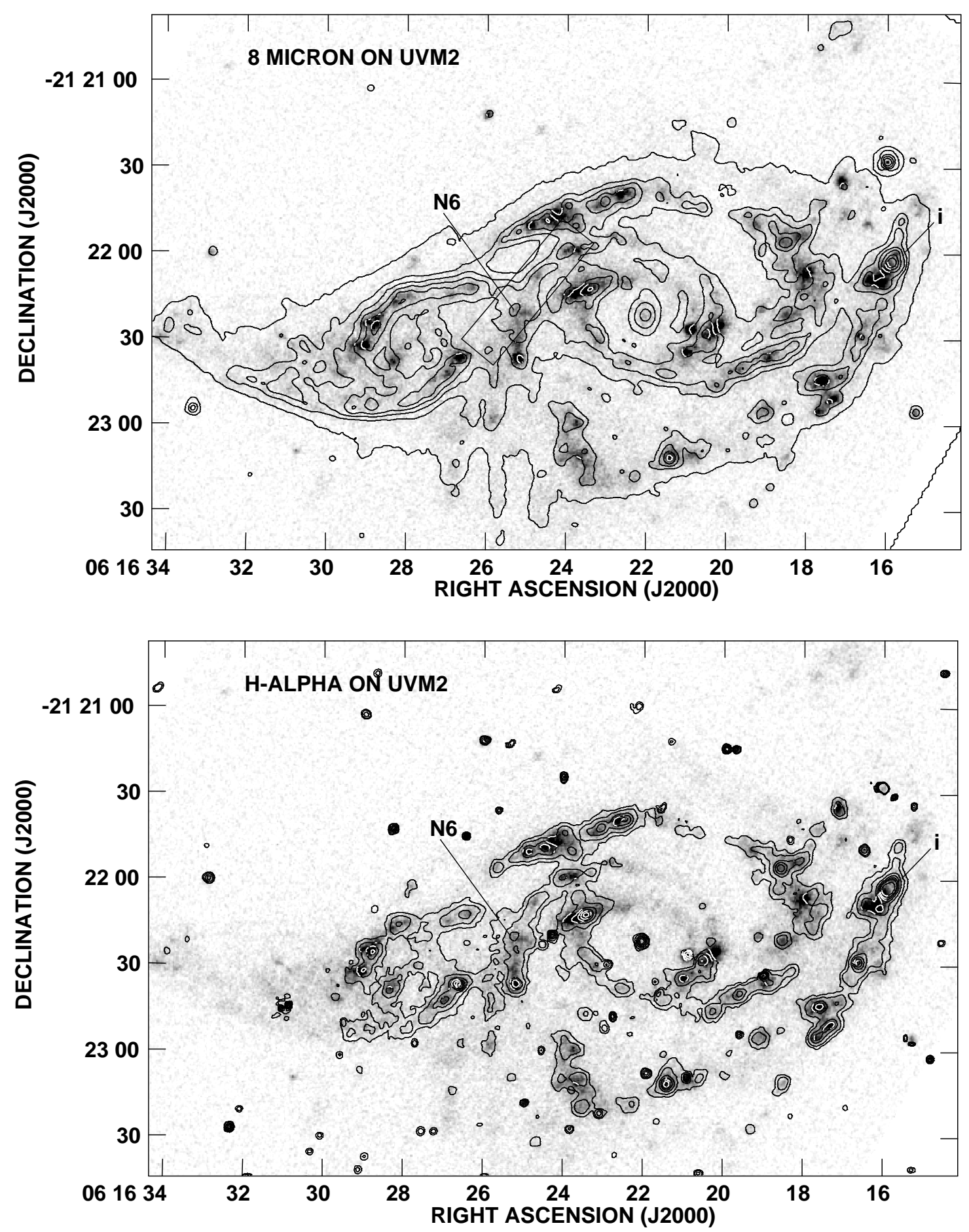

FIG. 3.- Top: Spitzer $8 \mu \mathrm{m}$ contours overlaid on the XMM-Newton-OM $U V M 2$ image. The contour levels are $0.5,2,4,8,16,32$, and $64 \mathrm{MJy} \mathrm{sr}^{-1}$. The long tilted box marks the location of the NE radio ridge. Bottom: Continuum-subtracted H $\alpha$ contours over the $U V M 2$ image. 


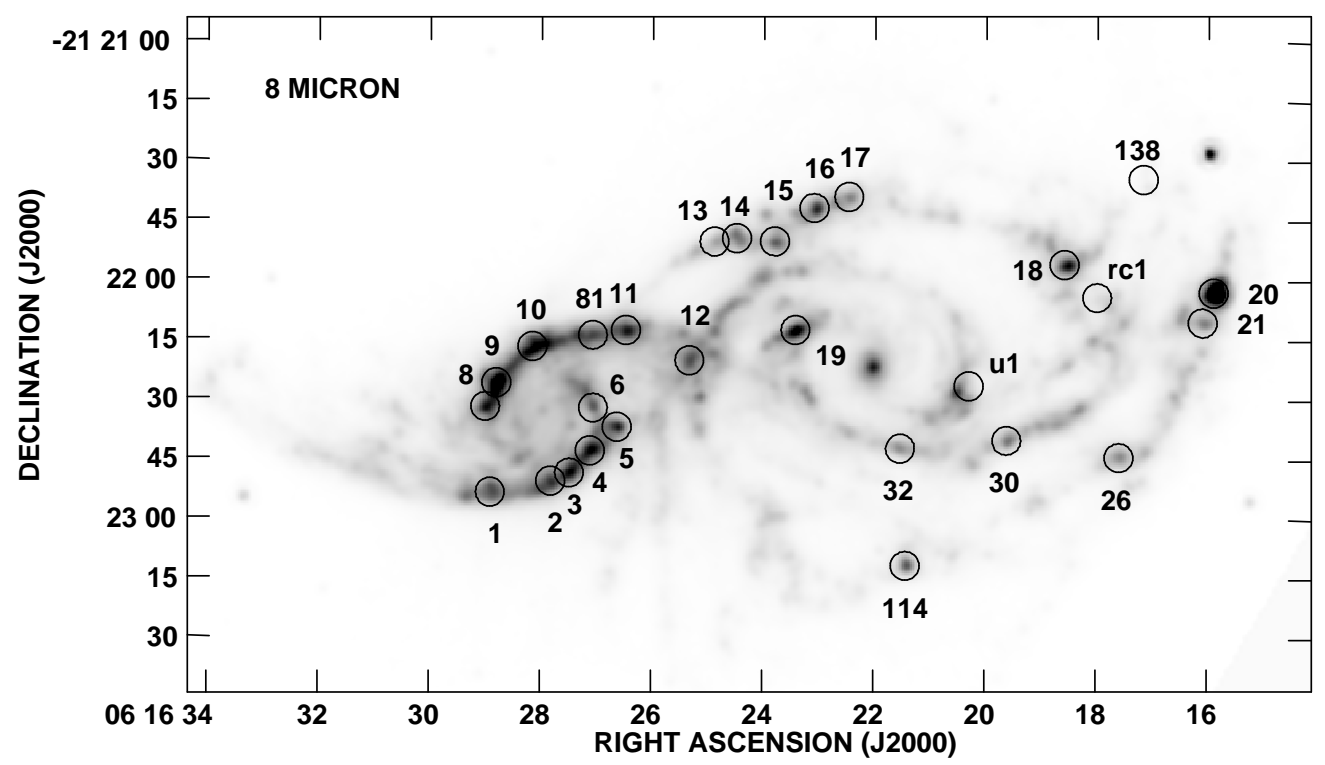

FIG. 4.- Spitzer $8 \mu \mathrm{m}$ image with numbers from Table 2 labelling the measured clumps. The $3.6^{\prime \prime}$ radius of each circle is the aperture radius.

TABLE 1

XMM-Newton Observations OF NGC 2207/IC 2163 ON 2005 August 31

\begin{tabular}{lllll}
\hline \hline Instrument & mode/filter & T-start $^{\mathrm{a}}$ & T-stop $^{\mathrm{a}}$ & $\mathrm{T}_{\text {exp }}{ }^{\mathrm{b}}$ \\
\hline EPIC pn & Ext. FF & $07: 14: 11$ & $20: 36: 01$ & 47640 \\
EPIC MOS-1 & FF & $06: 13: 14$ & $20: 25: 41$ & 51567 \\
EPIC MOS-2 & FF & $06: 13: 14$ & $20: 36: 01$ & 51572 \\
OM & U & $06: 17: 52$ & $07: 37: 32$ & 4460 \\
OM & U & $07: 37: 38$ & $08: 57: 28$ & 4479 \\
OM & B & $08: 57: 24$ & $10: 23: 15$ & 4840 \\
OM & B & $10: 23: 16$ & $11: 43: 01$ & 4460 \\
OM & UVW1 & $11: 43: 02$ & $13: 02: 47$ & 4460 \\
OM & UVW1 & $13: 02: 48$ & $14: 22: 23$ & 4460 \\
OM & UVM2 & $14: 22: 34$ & $15: 42: 19$ & 4460 \\
OM & UVM2 & $15: 42: 20$ & $17: 02: 05$ & 4460 \\
OM & UVM2 & $17: 02: 06$ & $18: 21: 51$ & 4479 \\
& & & &
\end{tabular}

a Start and End times are given in UT

b Observing time given in $\mathrm{s}$ 

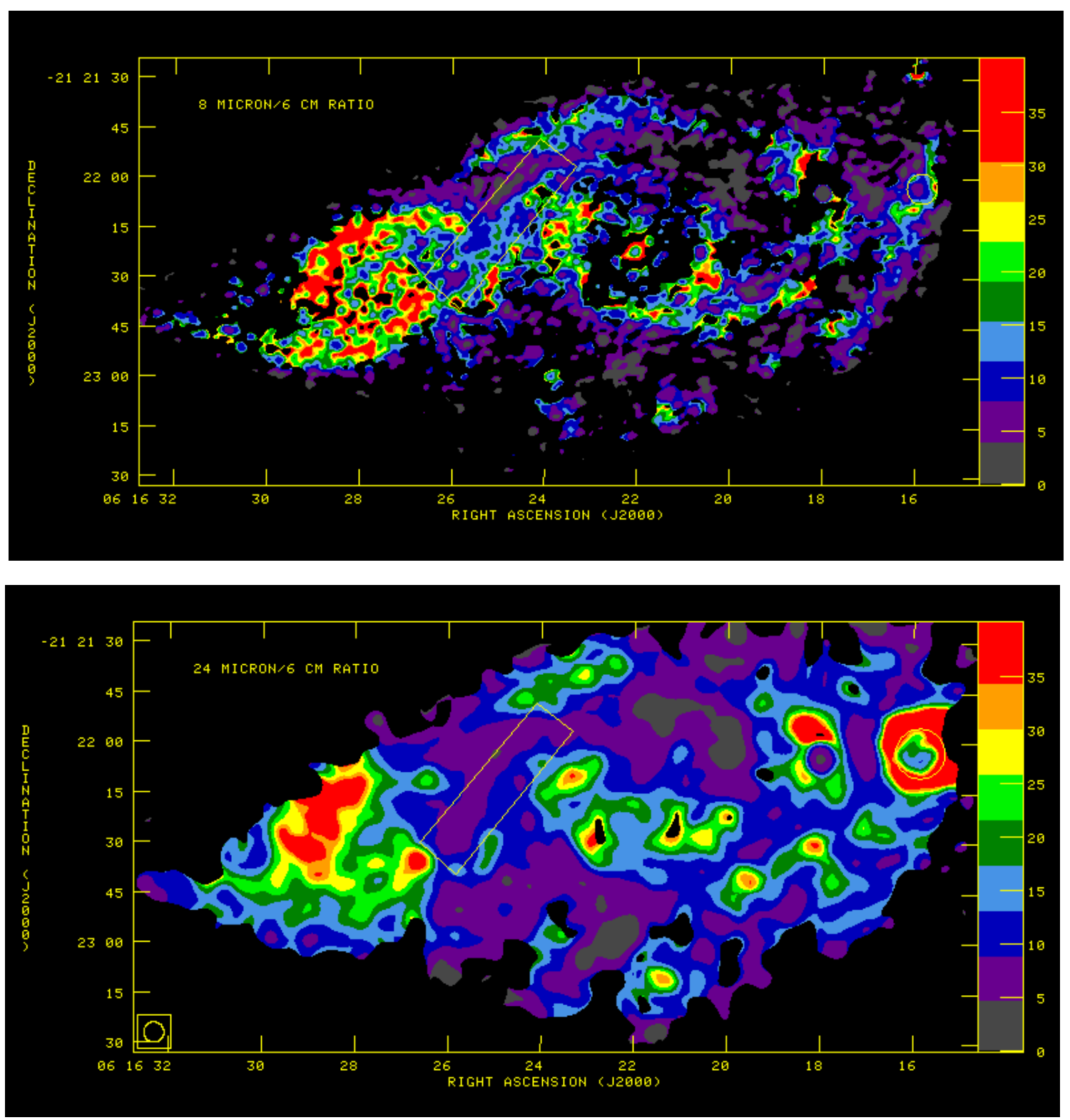

FIG. 5.- Top: Surface brightness ratio of Spitzer $8 \mu \mathrm{m}$ to $\lambda 6 \mathrm{~cm}$ radio continuum emission. Bottom: Surface brightness ratio of Spitzer $24 \mu \mathrm{m}$ to $\lambda 6 \mathrm{~cm}$ radio continuum emission. The long tilted box marks the location of the $N E$ radio ridge, and the circle marks the location of feature $i$. For the M81 HII regions, the mean value of $S_{\nu}(8 \mu \mathrm{m}) / S_{\nu}(6 \mathrm{~cm})$ is $19 \pm 5$, and the mean value of $S_{\nu}(24 \mu \mathrm{m}) / S_{\nu}(6 \mathrm{~cm})$ is $36 \pm 9$. In comparison the values of $I_{\nu}(8 \mu \mathrm{m}) / I_{\nu}(6 \mathrm{~cm})$ on the eyelids of IC 2163 are high and the values of $I_{\nu}(24 \mu \mathrm{m}) / I_{\nu}(6 \mathrm{~cm})$ on the $N E$ radio ridge are low. 

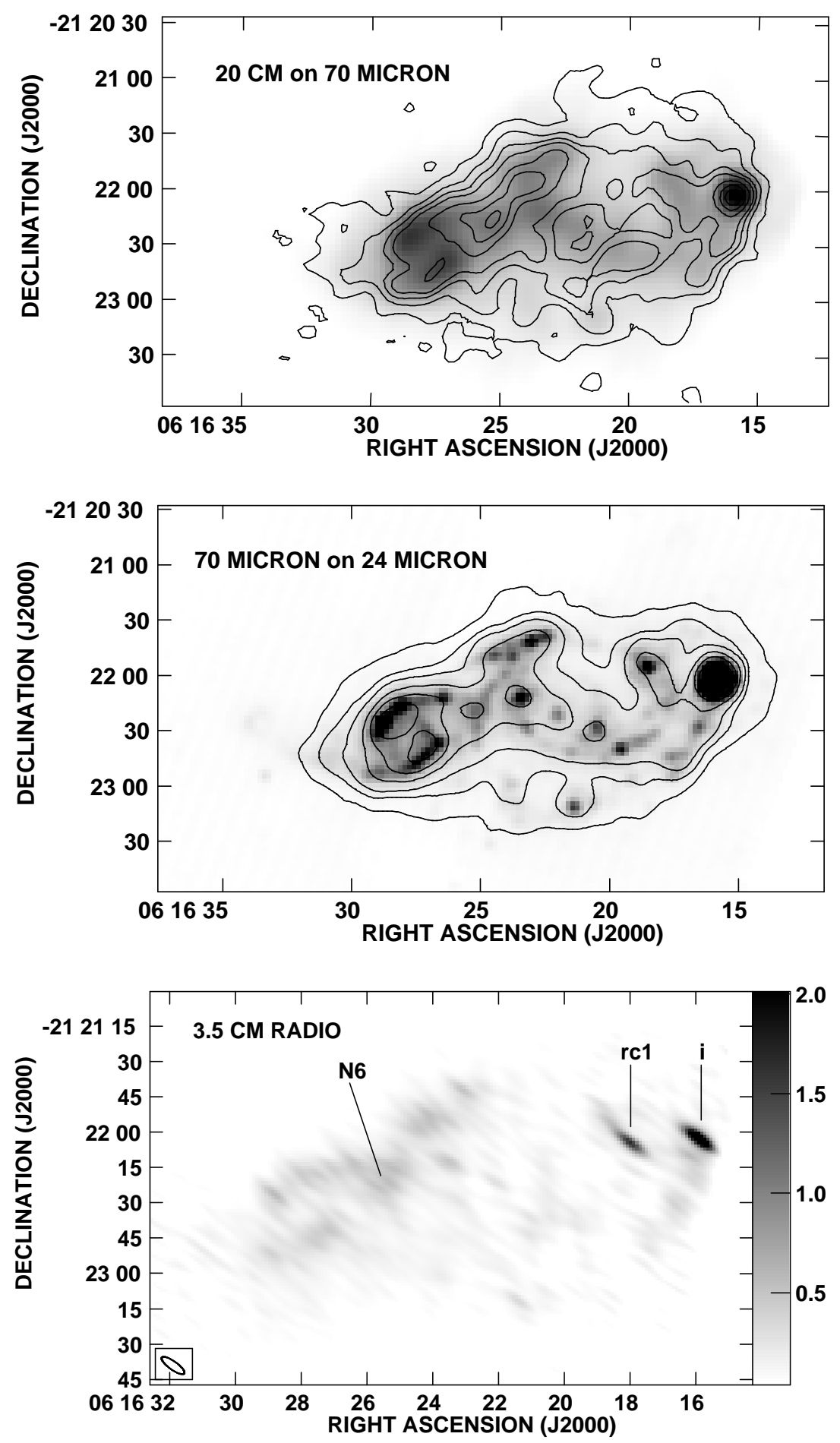

FIG. 6. - Top: Contours of radio continuum emission at $\lambda 20 \mathrm{~cm}$ (with $13.5^{\prime \prime} \times 12^{\prime \prime}$ resolution) overlaid on the Spitzer $70 \mu \mathrm{m}$ image (with $18^{\prime \prime}$ resolution). Contour levels at $1,2,3,4,6,8,10$ times $10 \mathrm{~K}$. Middle: Contours of $70 \mu \mathrm{m}$ emission overlaid on the Spitzer $24 \mu \mathrm{m}$ image. The contour levels are at 20,30, 40,60, 80,100,120,140 $\mathrm{MJy} \mathrm{sr}^{-1}$. Bottom: Radio continuum emission at $\lambda 3.5 \mathrm{~cm}\left(\right.$ with $11.7^{\prime \prime} \times 3.5^{\prime \prime}$ resolution). The eyelids are brighter than the $N E$ radio ridge at $24 \mu \mathrm{m}$ and $70 \mu \mathrm{m}$, whereas the $N E$ radio ridge is clearly brighter than the eyelids in the radio continuum at $\lambda 20 \mathrm{~cm}$. In this $\lambda 3.5 \mathrm{~cm}$ image from observations in $2003, \mathrm{rc} 1$ is much brighter than every source in the galaxy pair except feature $i$. 

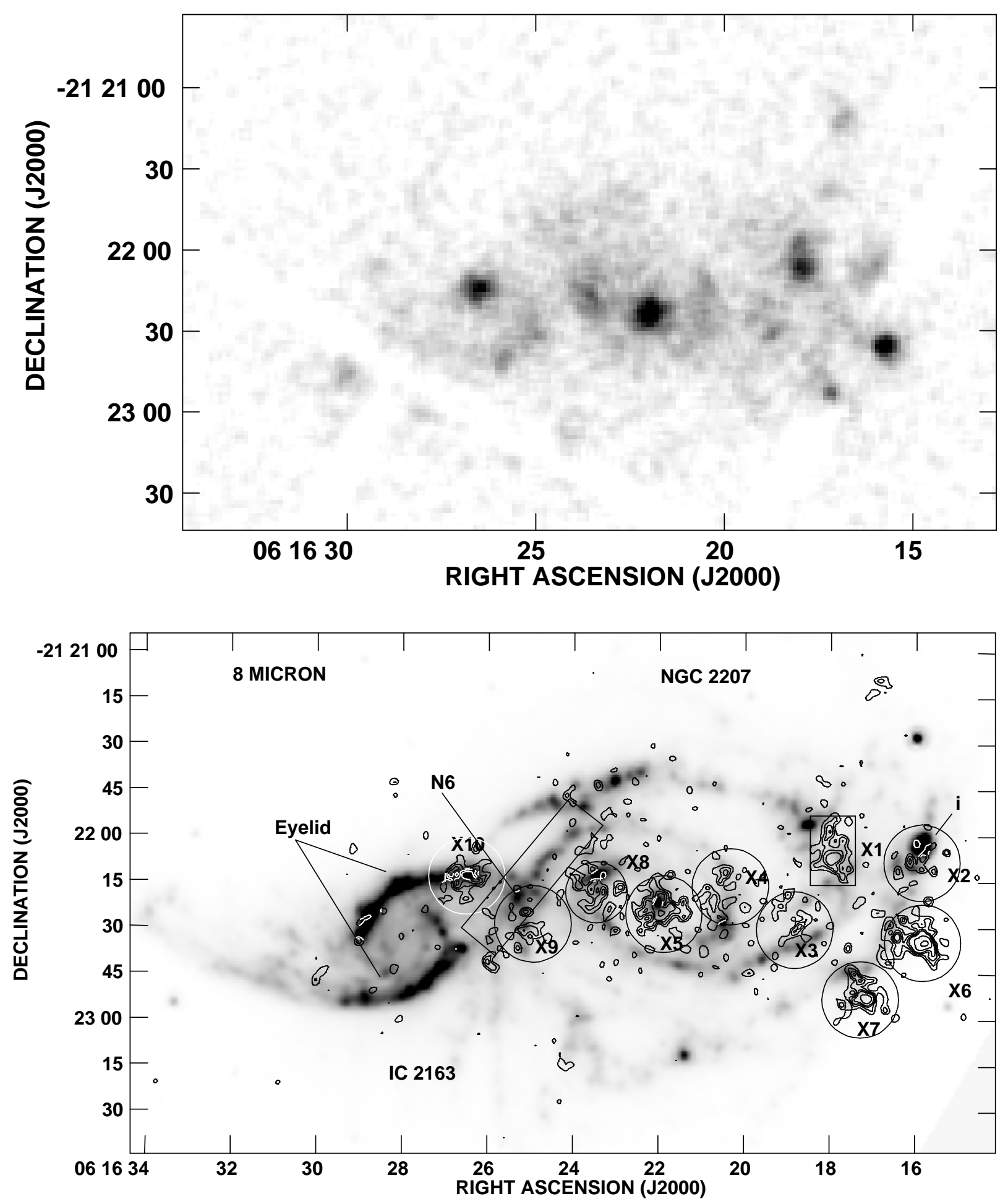

FIG. 7.- Top: X-ray image of the combined, smoothed, screened EPIC pn and MOS data in the $0.5-10.0$ keV energy range. The gaps result from including the pn camera data in this image. Bottom: $8 \mu \mathrm{m}$ Spitzer image with X-ray contours from the MOS data only overlaid. The entire field of interest fits onto a single CCD chip in the MOS camera data. The sources refer to Table 7 The circles and boxes mark the source extraction regions for the X-ray analysis. For the X-ray contours, the MOS data with FWHM of the PSF $\sim 5^{\prime \prime}$ was not smoothed, and the small-scale wiggles in the contours are generally not significant. The X-ray image used in the bottom panel has better spatial resolution but poorer sensitivity than the X-ray image in the top panel. 

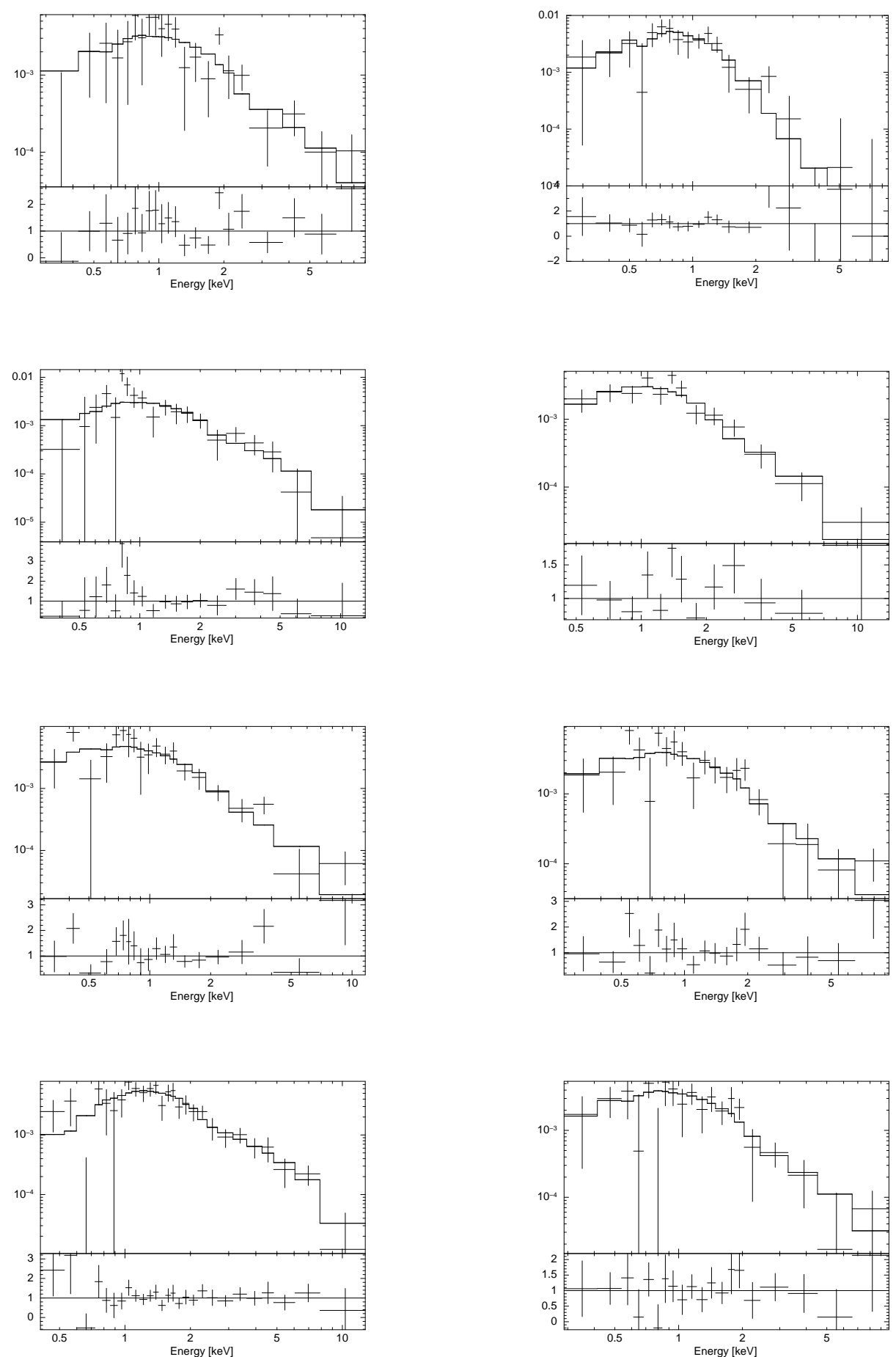

FIG. 8.- X-ray EPIC pn spectra of sources labelled in Figure 7 and listed in Table 7 The spectra were fitted with an absorbed power law model with Galactic and intrinsic absorption as listed in Table7 From top to bottom, the spectra in the left column are for sources X1, $\mathrm{X} 4, \mathrm{X} 8$, and $\mathrm{X} 10$ and the spectra in the right column are for sources $\mathrm{X} 3, \mathrm{X} 6, \mathrm{X} 9$, and the NE radio ridge. The ordinate on each spectrum is the normalized counts $\mathrm{s}^{-1} \mathrm{kev}^{-1}$ and the plot below each spectrum is the ratio of observed-to-model counts. 

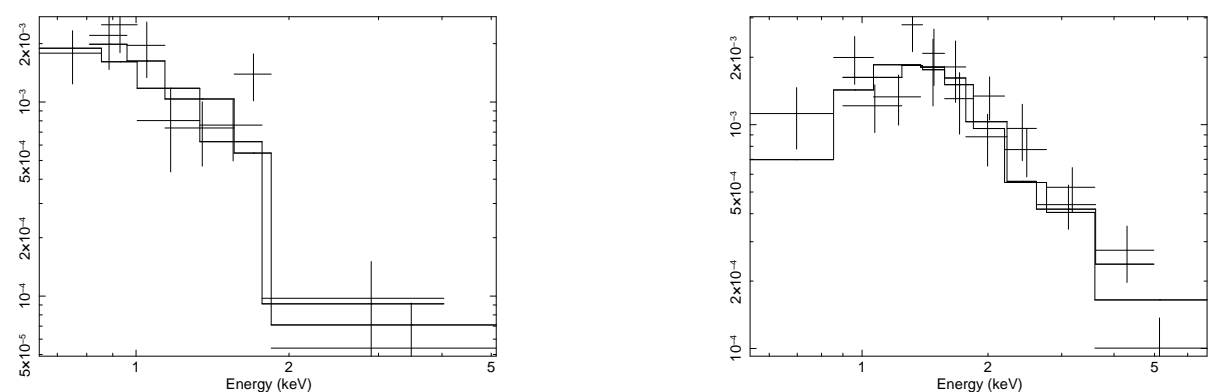

FIG. 9.- X-ray MOS spectra of sources X2 (left panel) and X7 (right panel). These lie at the chip edge in the EPIC pn observations.
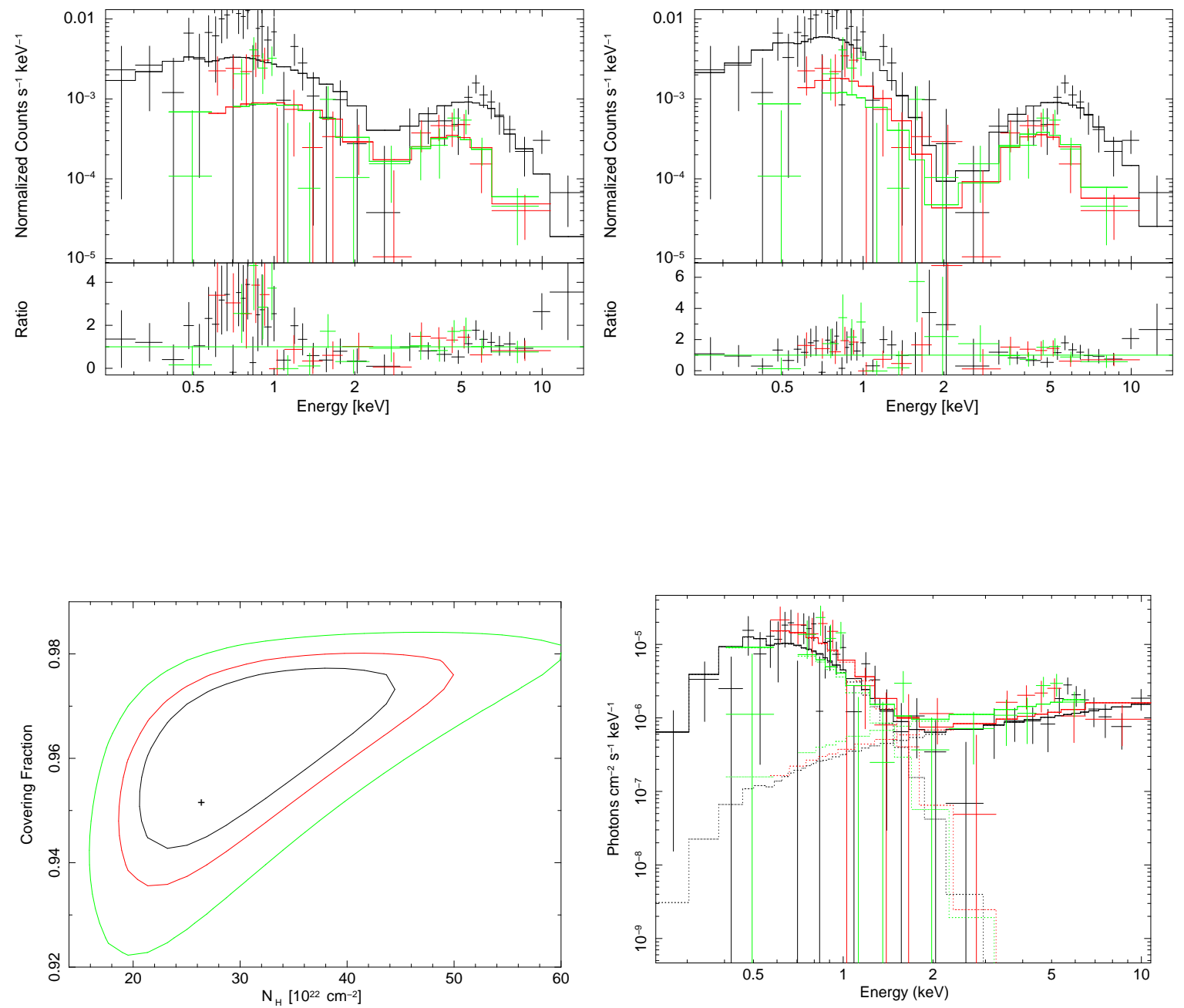

FIG. 10.- EPIC pn (black) and MOS 1 and 2 (red and green) spectra of the nucleus of NGC 2207 fitted by a power law model with partial covering absorber (upper left panel) and blackbody plus absorbed power law spectrum (upper right panel). Note the hard X-ray emission at $5-10 \mathrm{keV}$. Both fits include absorption by neutral gas in the Milky Way with an absorption column density of $1.13 \times 10^{21}$ $\mathrm{cm}^{-2}$ (Dickev \& Lockman 1990). The lower left panel displays the contour plot for the absorption column density and covering fraction of the partial-covering absorber model. The lower right panel shows the blackbody component and the power-law component separately for the model in the upper right panel. 

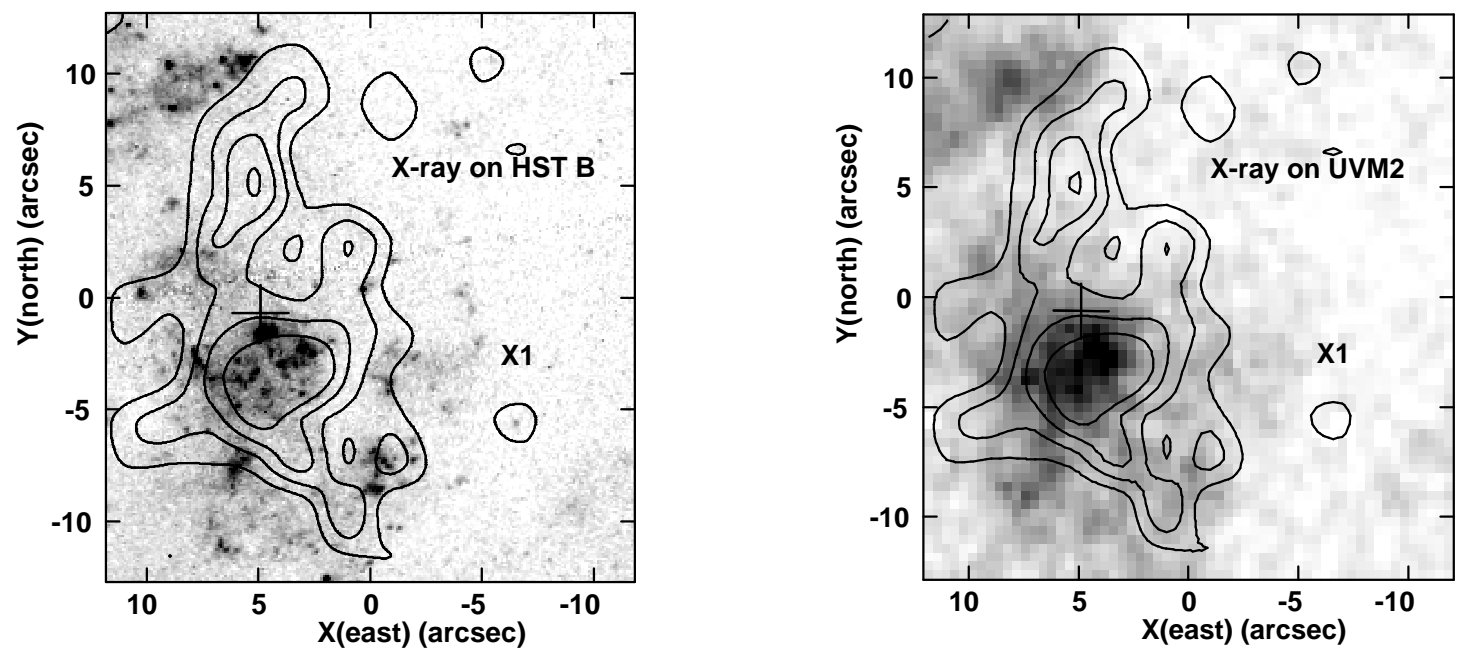

FIG. 11. - For source X1, X-ray contours from the MOS data overlaid on the HST B-band image (left panel) and on the $U V M 2$ image (right panel). The location of the unresolved radio clump $\mathrm{rc} 1$ is marked by a plus sign with width equal to the HPBW of the $\lambda 6 \mathrm{~cm}$ synthesized beam.

TABLE 2

IMAGES OF NGC $2207 /$ IC 2163

\begin{tabular}{lcl}
\hline \hline \multicolumn{1}{c}{ Image } & FWHM of PSF & \multicolumn{1}{c}{ Reference } \\
\hline$H S T$ B WFPC2 & $\sim 0.18^{\prime \prime}$ a & Elmegreen et al. (2001) \\
$U V M 2$ & $1.8^{\prime \prime}$ & this paper \\
$U V W 1$ & $2.0^{\prime \prime}$ & this paper \\
MOS X-ray & $\sim 5^{\prime \prime}$ & this paper \\
MOS + PN X-ray & $\sim 9^{\prime \prime}$ & this paper \\
Spitzer $8 \mu \mathrm{m}$ & $2.4^{\prime \prime}$ & Elmegreen et al. $(2006)$ \\
Spitzer $24 \mu \mathrm{m}$ & $6^{\prime \prime}$ & Elmegreen et al. (2006) \\
Spitzer $70 \mu \mathrm{m}$ & $18^{\prime \prime}$ & Elmegreen et al. (2006) \\
Spitzer $160 \mu \mathrm{m}$ & $40^{\prime \prime}$ & Elmegreen et al. (2006) \\
$\lambda 3.5 \mathrm{~cm}$ radio continuum & $11.7^{\prime \prime} \times 3.5^{\prime \prime}$ & VLA public archives \\
$\lambda 6 \mathrm{~cm}$ radio continuum & $2.48^{\prime \prime} \times 1.3^{\prime \prime}$ & this paper \\
$\lambda 6 \mathrm{~cm}$ radio continuum & $2.5^{\prime \prime}$ & this paper \\
$\lambda 6 \mathrm{~cm}$ radio continuum & $6^{\prime \prime}$ & this paper \\
$\lambda 20 \mathrm{~cm}$ radio continuum & $13.5^{\prime \prime} \times 12^{\prime \prime}$ & Elmegreen et al. (1995a) \\
$\mathrm{H} \mathrm{I}$ & $13.5^{\prime \prime} \times 12^{\prime \prime}$ & Elmegreen et al. (1995a) \\
SEST ${ }^{12} \mathrm{CO} J=1 \rightarrow 0$ & $43^{\prime \prime}$ & Thomasson (2004) \\
$\mathrm{H} \alpha$ & $4.2^{\prime \prime} \times 3.6^{\prime \prime}$ & Elmegreen et al. (2001) \\
& &
\end{tabular}

$\mathrm{a} \sim 0.18^{\prime \prime}$ for wide field and $\sim 0.09^{\prime \prime}$ for planetary camera 

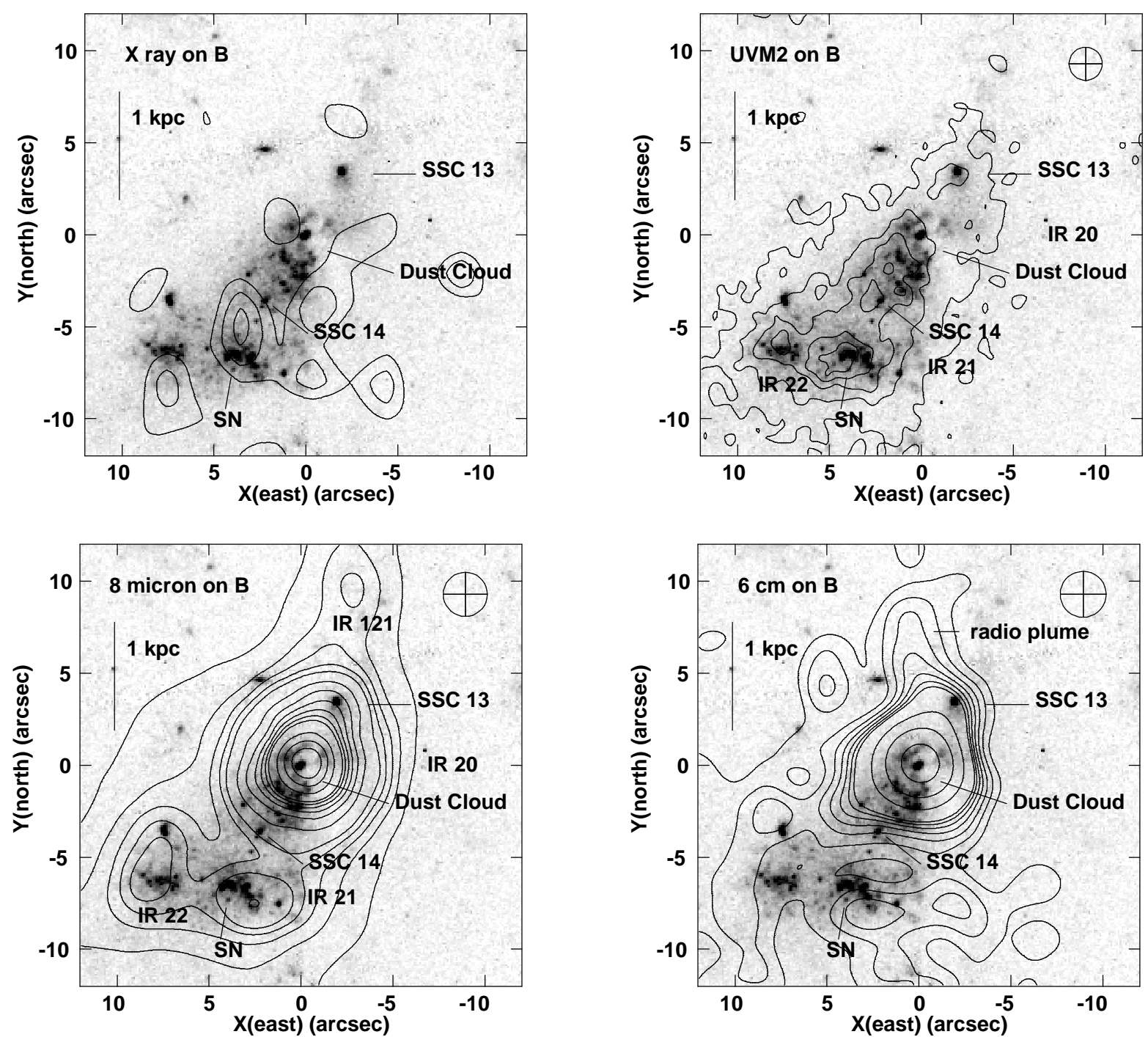

FIG. 12.- Overlays on the HST B-image of feature $i$ at different wavelengths. The upper left panel displays the contours of the X-ray emission, the upper right the UVM2 data from the XMM-Newton OM observations, the lower left the $8 \mu \mathrm{m}$ Spitzer observations, and the lower right the $\lambda 6 \mathrm{~cm}$ radio continuum emission. The $8 \mu \mathrm{m}$ contours are at $2 \ldots(2) \ldots 10 \ldots(5) \ldots 40,60,80$ and $100 \mathrm{MJy} \mathrm{sr}^{-1}$. The $\lambda 6 \mathrm{~cm}$ radio contours are at $4 \ldots(2) \ldots 16,32,64,128$ times the rms noise of $0.016 \mathrm{mJy}^{-}$beam ${ }^{-1}$. The label SN points to a stellar image at the SW edge of the $r=0.3^{\prime \prime}$ error circle that Van Dyk et al. (2003) give for the position of SN 1999ec; they conclude that this star is too bright to be the progenitor. 
TABLE 3

Spitzer and Ultraviolet Clumps in NGC 2207/IC 2163

\begin{tabular}{|c|c|c|c|c|c|c|c|c|}
\hline Clump $^{a}$ & $\begin{array}{c}S_{\nu}(6 \mathrm{~cm}) \\
(\mathrm{mJy})\end{array}$ & $\begin{array}{c}S_{\nu}(8 \mu \mathrm{m}) \\
(\mathrm{mJy})\end{array}$ & $(8 \mu \mathrm{m} / 6 \mathrm{~cm})^{\mathrm{b}}$ & $S(\mathrm{H} \alpha)^{\mathrm{c}}$ & $\begin{array}{c}A_{v}^{\mathrm{d}} \\
(\mathrm{mag})\end{array}$ & $\begin{array}{l}U V M 2 \\
(\mathrm{mag})\end{array}$ & $(8 \mu \mathrm{m} / U V M 2)^{\mathrm{e}}$ & $\begin{array}{c}U V M 2-U V W 1 \\
(\mathrm{mag})\end{array}$ \\
\hline IR 1 & 0.51 & 10.4 & 21 & 0.59 & 6.1 & 18.6 & 370 & 0.49 \\
\hline IR 2 & 0.36 & 11.9 & 33 & 0.24 & 6.9 & $>19.2$ & $>710$ & $>0.00$ \\
\hline IR 3 & 0.63 & 13.5 & 21 & 0.69 & 6.2 & 19.0 & 650 & 0.58 \\
\hline IR 4 & 0.39 & 12.5 & 32 & 2.6 & 3.7 & 17.7 & 190 & 0.38 \\
\hline IR 5 & 0.11 & 8.2 & 72 & 4.4 & 1.2 & 16.9 & 62 & 0.10 \\
\hline IR 8 & 0.22 & 10.3 & 47 & 0.82 & 4.4 & 16.6 & 59 & 0.06 \\
\hline IR 9 & 0.75 & 19.3 & 26 & 2.4 & 4.7 & 16.7 & 120 & 0.17 \\
\hline IR 10 & 0.34 & 15.0 & 44 & 1.5 & 4.3 & 17.4 & 170 & 0.24 \\
\hline IR 11 & 0.46 & 11.2 & 25 & 2.0 & 4.3 & 18.0 & 220 & 0.13 \\
\hline IR 81 & 0.41 & 9.6 & 23 & 1.9 & 4.1 & 18.4 & 280 & -0.23 \\
\hline IR 6 & 0.10 & 5.5 & 57 & $\ldots$ & $\ldots$ & $>19.2$ & $>330$ & $>0.59$ \\
\hline IR 13 & 0.34 & 5.0 & 15 & 1.7 & 4.1 & 16.8 & 34 & 0.03 \\
\hline IR 14 & 0.42 & 6.9 & 17 & 2.7 & 3.7 & 16.7 & 42 & 0.10 \\
\hline IR 15 & 0.41 & 4.7 & 11 & 0.88 & 5.2 & 17.4 & 56 & 0.06 \\
\hline IR 16 & 0.65 & 9.4 & 14 & 2.9 & 4.2 & 17.3 & 100 & 0.21 \\
\hline IR 17 & 0.56 & 6.2 & 11 & 3.9 & 3.6 & 16.9 & 43 & 0.01 \\
\hline IR 18 & 0.50 & 9.8 & 20 & 3.4 & 3.6 & 17.3 & 100 & 0.21 \\
\hline IR 19 & 0.53 & 11.5 & 21 & 4.7 & 3.3 & 16.4 & 53 & -0.01 \\
\hline $\operatorname{IR} 12^{\mathrm{f}}$ & 0.81 & 6.1 & 7.3 & 1.1 & 5.9 & 17.8 & 100 & 0.35 \\
\hline IR $20^{\mathrm{g}}$ & 4.39 & 35.1 & 8.0 & 11.6 & 4.9 & 16.8 & 240 & 0.23 \\
\hline IR $21^{\mathrm{h}}$ & 0.28 & 5.3 & 19 & 1.9 & 3.6 & 16.3 & 22 & -0.09 \\
\hline IR 26 & 0.27 & 5.7 & 21 & 2.1 & 3.5 & 16.5 & 28 & -0.07 \\
\hline IR 30 & 0.25 & 5.9 & 24 & 1.8 & 3.5 & 17.8 & 95 & 0.14 \\
\hline IR 32 & 0.23 & 4.8 & 21 & $\ldots$ & $\ldots$ & $>19.2$ & $>290$ & $>0.14$ \\
\hline IR 114 & 0.34 & 5.7 & 17 & 3.2 & 3.2 & 17.0 & 43 & -0.01 \\
\hline u1 & 0.13 & 5.6 & 42 & $\ldots$ & $\ldots$ & 16.2 & 22 & -0.03 \\
\hline IR 138 & $<0.04$ & 0.81 & $\ldots$ & $\ldots$ & $\ldots$ & 17.0 & 6.2 & -0.25 \\
\hline rc1 & 0.90 & 1.4 & 1.6 & $\ldots$ & $\ldots$ & 16.8 & 8.9 & -0.10 \\
\hline
\end{tabular}

${ }^{a}$ Clumps are identified in Fig. 4. IR clump positions are listed in Elmegreen et al. (2006)

b $(8 \mu \mathrm{m} / 6 \mathrm{~cm})$ is the flux density ratio $S_{\nu}(8 \mu \mathrm{m}) / S_{\nu}(6 \mathrm{~cm})$.

c $\mathrm{S}(\mathrm{H} \alpha)$ in units of $10^{-14} \mathrm{erg} \mathrm{cm}^{-2} \mathrm{~s}^{-1}$

d These are upper limits obtained by assuming the radio continuum emission has no nonthermal component.

e $(8 \mu \mathrm{m} / U V M 2)$ is the flux density ratio $S_{\nu}(8 \mu \mathrm{m}) / S_{\nu}(U V M 2)$.

$\mathrm{f}$ in H I massive cloud N6 on the NE radio ridge of NGC 2207

g Feature $i$

h contains SN 1999ec

TABLE 4

H II REGIONS IN M81

\begin{tabular}{ccccccc}
\hline \hline H II Region $^{\mathrm{a}}$ & $\begin{array}{c}S_{\nu}(6 \mathrm{~cm}) \\
(\mathrm{mJy})\end{array}$ & $(8 \mu \mathrm{m} / 6 \mathrm{~cm})^{\mathrm{b}}$ & $(24 \mu \mathrm{m} / 6 \mathrm{~cm})^{\mathrm{c}}$ & $(8 \mu \mathrm{m} / N U V)^{\mathrm{d}}$ & $\begin{array}{c}A_{v}(\mathrm{PG}) \\
(\mathrm{mag})\end{array}$ & $\begin{array}{c}A_{v}(\mathrm{~K}) \\
(\mathrm{mag})\end{array}$ \\
\hline K181 & $1.10 \pm 0.07$ & $18 \pm 1$ & $52 \pm 3$ & 12 & 0.70 & $0.5 \pm 0.2$ \\
$\mathrm{~K} 178$ & $1.01 \pm 0.07$ & $15 \pm 1$ & $29 \pm 2$ & 31 & 1.33 & $1.2 \pm 0.2$ \\
$\mathrm{~K} 123$ & $0.87 \pm 0.12$ & $13 \pm 2$ & $25 \pm 3$ & 21 & 0.87 & $0.0 \pm 0.3$ \\
$\mathrm{~K} 152$ & $0.80 \pm 0.14$ & $15 \pm 3$ & $34 \pm 6$ & 15 & 0.42 & $0.6 \pm 0.3$ \\
$\mathrm{~K} 125$ & $0.74 \pm 0.14$ & $16 \pm 3$ & $30 \pm 6$ & 15 & 0.43 & $0.5 \pm 0.3$ \\
$\mathrm{~K} 159$ & $0.69 \pm 0.13$ & $19 \pm 4$ & $42 \pm 8$ & 17 & 1.01 & $1.0 \pm 0.3$ \\
$\mathrm{~K} 138$ & $0.60 \pm 0.09$ & $19 \pm 3$ & $48 \pm 7$ & 12 & 0.67 & $0.7 \pm 0.3$ \\
$\mathrm{~K} 172$ & $0.52 \pm 0.07$ & $20 \pm 3$ & $35 \pm 5$ & 9.0 & 0.73 & $-0.1 \pm 0.3$ \\
$\mathrm{~K} 156$ & $0.50 \pm 0.10$ & $21 \pm 4$ & $25 \pm 5$ & 48 & 1.91 & $2.1 \pm 0.4$ \\
$\mathrm{~K} 187$ & $0.45 \pm 0.08$ & $29 \pm 5$ & $38 \pm 7$ & 29 & 0.36 & $0.2 \pm 0.3$ \\
$\mathrm{~K} 102$ & $0.27 \pm 0.05$ & $28 \pm 5$ & $41 \pm 8$ & 23 & 0.88 & $0.9 \pm 0.3$ \\
& mean & $19 \pm 5$ & $36 \pm 9$ & $21 \pm 11$ & $0.85 \pm 0.46$ & $0.8 \pm 0.6$
\end{tabular}

a The radio continuum data and $A_{v}(\mathrm{~K})$ from Kaufman et al. (1987) and the Spitzer data, GALEX NUV data, and $A_{v}(\mathrm{PG})$ from Pérez-González et al. (2006)

b $(8 \mu \mathrm{m} / 6 \mathrm{~cm})$ is the flux density ratio $S_{\nu}(8 \mu \mathrm{m}) / S_{\nu}(6 \mathrm{~cm})$.

c $(24 \mu \mathrm{m} / 6 \mathrm{~cm})$ is the flux density ratio $S_{\nu}(24 \mu \mathrm{m}) / S_{\nu}(6 \mathrm{~cm})$.

d $(8 \mu \mathrm{m} / N U V)$ is the flux density ratio $S_{\nu}(8 \mu \mathrm{m}) / S_{\nu}(N U V)$.

e The uncertainty listed with the mean is the standard deviation $\sigma$ of the sample, not the measurement uncertainty or the standard deviation of the mean. 
TABLE 5

COMParison OF $q_{\text {IR }}$ VAlues

\begin{tabular}{lccccc}
\hline \hline \multicolumn{1}{c}{ Sample $^{\mathrm{a}}$} & $q_{F I R}$ & $q_{70}$ & $q_{24}$ & $q_{8}$ & $(24 \mu \mathrm{m} / 8 \mu \mathrm{m})^{\mathrm{b}}$ \\
\hline NGC 2207/IC 2163 & 1.81 & 1.79 & 0.71 & 0.49 & 1.6 \\
Spitzer First-Look: & $\ldots$ & $2.15 \pm 0.16$ & $0.94 \pm 0.23$ & $\ldots$ & $\ldots$ \\
Appleton et al. (2004) & $\ldots$ & $\ldots$ & $1.07 \pm 0.17$ & $0.91 \pm 0.13$ & $\ldots$ \\
Wu et al. (2005) & $\ldots$ & $2.39 \pm 0.28$ & $1.31 \pm 0.31$ & $1.12 \pm 0.26$ & $1.7 \pm 1.6$ \\
SINGS & $\ldots$ & $2.40 \pm 0.29$ & $1.33 \pm 0.31$ & $1.15 \pm 0.24$ & $1.5 \pm 1.1$ \\
SINGS & $\ldots$ & $\ldots$ & $\ldots$ & $\ldots$ & $\ldots$ \\
IRAS (Condon 1992) & $2.3 \pm 0.2$ & $\ldots$ & $1.50 \pm 0.10$ & $1.22 \pm 0.12$ & $1.9 \pm 0.5$ \\
M81 H II regions & $\ldots$ & & & & \\
& & & & \\
\hline
\end{tabular}

a The Spitzer First-Look, SINGS, and IRAS samples refer to the integrated emission from the entire galaxy. The M81 H II region sample refers to the H II regions in Table 4 The uncertainties listed are the standard deviations $\sigma$ of the samples.

b $(24 \mu \mathrm{m} / 8 \mu \mathrm{m})$ is the flux density ratio $S_{\nu}(24 \mu \mathrm{m}) / S_{\nu}(8 \mu \mathrm{m})$.

c excluding galaxies with poor quality data or low metallicity

$\mathrm{d}$ excluding galaxies with poor quality data or low metallicity and also omitting E and S0 galaxies 
TABLE 6

Integrated $\lambda 6$ CM Radio and Infrared Flux Densities

\begin{tabular}{lcccccc}
\hline \hline \multicolumn{1}{c}{ Region } & $\begin{array}{c}S_{\nu}(6 \mathrm{~cm}) \\
(\mathrm{mJy})\end{array}$ & $\begin{array}{c}S_{\nu}(8 \mu \mathrm{m}) \\
(\mathrm{mJy})\end{array}$ & $\begin{array}{c}S_{\nu}(24 \mu \mathrm{m}) \\
(\mathrm{mJy})\end{array}$ & $(8 \mu \mathrm{m} / 6 \mathrm{~cm})^{\mathrm{a}}$ & $(24 \mu \mathrm{m} / 6 \mathrm{~cm})^{\mathrm{b}}$ & $(24 \mu \mathrm{m} / 8 \mu \mathrm{m})^{\mathrm{c}}$ \\
\hline NE radio ridge box & $13.2 \pm 0.2$ & 121 & $112^{\mathrm{d}}$ & 9.1 & $8.5^{\mathrm{d}}$ & 23 \\
eyelid box & $21.7 \pm 0.3$ & 409 & 506 & 19 & 15 & $0.9^{\mathrm{d}}$ \\
combined galaxies & $132 \pm 1$ & $1.22 \times 10^{3}$ & $2.00 \times 10^{3}$ & 9.3 & 1.2 & 1.6 \\
M81 H II regions & $\cdots$ & $\cdots$ & $\cdots$ & $19 \pm 5$ & $36 \pm 9$ \\
\hline
\end{tabular}

a $(8 \mu \mathrm{m} / 6 \mathrm{~cm})$ is the flux density ratio $S_{\nu}(8 \mu \mathrm{m}) / S_{\nu}(6 \mathrm{~cm})$.

b $(24 \mu \mathrm{m} / 6 \mathrm{~cm})$ is the flux density ratio $S_{\nu}(24 \mu \mathrm{m}) / S_{\nu}(6 \mathrm{~cm})$.

c $(24 \mu \mathrm{m} / 8 \mu \mathrm{m})$ is the flux density ratio $S_{\nu}(24 \mu \mathrm{m}) / S_{\nu}(8 \mu \mathrm{m})$.

d uncertain because the $14^{\prime \prime}$ width of the $N E$ radio ridge box is only $2.3 \times$ the FWHM of the $24 \mu \mathrm{m}$ PSF.

TABLE 7

X-ray spectral analysis of X-ray sources displayed in Figure $7^{2}$

\begin{tabular}{|c|c|c|c|c|c|c|c|c|}
\hline Source & $\mathrm{RA}_{2000^{\mathrm{b}}}$ & $\operatorname{Dec}_{2000}{ }^{c}$ & $N_{\mathrm{H}, \text { intr }}{ }^{\mathrm{d}}$ & $\alpha_{\mathrm{X}}^{\mathrm{e}}$ & $k T^{\mathrm{f}}$ & $\chi^{2} /$ dof & $F_{0.3-10.0^{\mathrm{g}}}$ & $N(\mathrm{HI})^{\mathrm{h}}$ \\
\hline $\mathrm{X} 1$ & $\begin{array}{llll}06 & 16 & 17.94\end{array}$ & -212204.5 & $0.12_{-0.12}^{+0.21}$ & $1.06_{-0.59}^{+0.47}$ & $\cdots$ & $20 / 19$ & 4.70 & $0.32 \pm 0.05$ \\
\hline $\mathrm{X} 2$ & $\begin{array}{lll}06 & 16 & 15.86\end{array}$ & -212208.5 & 0.00 & $2.17_{-0.56}^{+1.62}$ & $\ldots$ & $10 / 6$ & 5.40 & $0.32 \pm 0.08$ \\
\hline $\mathrm{X} 3$ & $\begin{array}{lll}06 & 16 & 18.83\end{array}$ & -212230.5 & $0.03_{-0.04}^{+0.04}$ & $\ldots$ & $2.12_{-0.68}^{+0.68}$ & $16 / 16$ & 1.39 & $0.28 \pm 0.05$ \\
\hline $\mathrm{X} 4$ & $06 \quad 16 \quad 20.34$ & $-2122 \quad 16.5$ & $0.12_{-0.12}^{+0.21}$ & $0.93_{-0.50}^{+0.49}$ & $\ldots$ & $16 / 17$ & 4.03 & $0.12 \pm 0.03$ \\
\hline $\mathrm{X} 5^{\mathrm{i}}$ & 061621.89 & -212225.5 & - & 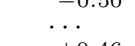 & $\ldots$ & $\ldots$ & $\ldots$ & $0.14 \pm 0.04$ \\
\hline $\mathrm{X} 6$ & $\begin{array}{lll}06 & 16 & 15.84\end{array}$ & -212234.6 & $0.15_{-0.13}^{+0.15}$ & $0.97_{-0.30}^{+0.46}$ & $\ldots$ & $11 / 10$ & 8.82 & $0.23 \pm 0.11$ \\
\hline $\mathrm{X} 7$ & $\begin{array}{lll}06 & 16 & 17.29\end{array}$ & -212253.2 & $0.27_{-0.24}^{+0.42}$ & $0.86_{-0.41}^{+0.58}$ & $\ldots$ & $20 / 13$ & 10.4 & $0.27 \pm 0.10$ \\
\hline $\mathrm{X} 8$ & $\begin{array}{lll}06 & 16 & 23.47\end{array}$ & $-2122 \quad 18.5$ & $0.03_{-0.03}^{+0.12}$ & $1.13_{-0.13}^{+0.51}$ & $\ldots$ & $20 / 17$ & 4.30 & $0.27 \pm 0.10$ \\
\hline X9 & 061624.95 & -212228.9 & $0.04_{-0.04}^{+0.15}$ & $1.05_{-0.43}^{+0.63}$ & $\ldots$ & $15 / 17$ & 3.44 & $0.41 \pm 0.06$ \\
\hline $\mathrm{X} 10$ & 061626.50 & -212213.4 & $0.35_{-0.15}^{+0.27}$ & $1.03_{-0.27}^{+0.40}$ & $\ldots$ & $18 / 24$ & 9.80 & $0.44 \pm 0.08$ \\
\hline $\mathrm{RR}^{\mathrm{j}}$ & $\begin{array}{lll}06 & 16 & 25.13\end{array}$ & $-2122 \quad 23.4$ & $\begin{array}{l}0.09_{-0.09}^{+0.19} \\
0\end{array}$ & $1.14_{-0.55}^{+0.83}$ & $\ldots$ & $10 / 17$ & 3.85 & $\ldots$ \\
\hline
\end{tabular}

${ }^{a}$ For all sources the EPIC pn data were used except for X5 (NGC 2207 nucleus) for which the pn plus MOS data were fitted simultaneously in XSPEC (as shown in Figure10), and sources X2 and X7 for which only the MOS data were used because both sources were in a gap between two CCDs in the pn. All fits include absorption by neutral gas in our Galaxy with an absorption column density $N_{\mathrm{H} \text {,gal }}=1.13 \times 10^{21} \mathrm{~cm}^{-2}$ (Dickey \& Lockman 1990).

b RA in $h \mathrm{~ms}$

c Dec in ${ }^{\circ}$, , and $"$

d Intrinsic absorption column density at the location of NGC $2207 /$ IC 2163 ( $\mathrm{z}=0.00941)$ in units of $10^{22} \mathrm{~cm}^{-2}$.

e Energy spectral index $\alpha_{\mathrm{X}}$ for a single, absorbed, power-law model

f $k T$ in units of keV for Raymond-Smith plasma

$\mathrm{g}$ Flux in $0.3-10.0 \mathrm{keV}$ band corrected for Galactic and intrinsic absorption, in units of $10^{-14} \mathrm{ergs} \mathrm{s}^{-1} \mathrm{~cm}^{-2}$

h $N(\mathrm{HI})$ of the galaxy pair in units of $10^{22}$ atom $\mathrm{cm}^{-2}$ from the 21-cm line data of Elmegreen et al. (1995a)

${ }^{\mathrm{i}} \mathrm{X} 5=$ nucleus of NGC 2207. The simple absorbed power law model does not represent the data. More complicated spectral models are listed in Table 8

$\mathrm{j} \mathrm{RR}$ is the $N E$ radio ridge, not a discrete source 
TABLE 8

X-ray Spectral fits to the NGC 2207 nucleus $(X 5)^{a}$

\begin{tabular}{lcccccc}
\hline \hline XSPEC Model & $\alpha_{\mathrm{X}}^{\mathrm{b}}$ & $N_{\mathrm{H}, \mathrm{pc}}{ }^{\mathrm{c}}$ & $f_{\mathrm{pc}}$ & $\mathrm{kT}^{\mathrm{d}}$ & $\chi^{2} / \mathrm{dof}$ & $F_{0.3-10.0 \mathrm{keV}}{ }^{\mathrm{e}}$ \\
\hline zpcfabs ${ }^{*}$ powl & 1.00 (fixed) & $26.5_{-6.0}^{+5.5}$ & $0.95_{-0.01}^{+0.02}$ & $\ldots$ & $83 / 65$ & 20.6 \\
$\mathrm{bb}+$ powl & $-1.56_{-0.16}^{+0.14}$ & $\cdots$ & $\cdots$ & $163_{-19}^{+29}$ & $74 / 62$ & 13.5 \\
& & & & & & \\
\hline
\end{tabular}

a All fits include absorption by neutral gas in our Galaxy with an absorption column density $N_{\mathrm{H}, \text { gal }}=1.13 \times 10^{21} \mathrm{~cm}^{-2}$ (Dickev \& Lockman 1990).

b Energy spectral index $\alpha_{\mathrm{X}}$

c Intrinsic absorption column density at the location of NGC 2207/IC 2163 ( $\mathrm{z}=0.00941$ ) in units of $10^{22} \mathrm{~cm}^{-2}$.

d Blackbody temperature $\mathrm{kT}$ in units of $\mathrm{eV}$

e $0.3-10.0 \mathrm{keV}$ flux corrected for Galactic and intrinsic absorption, in units of $10^{-14} \mathrm{ergs}$ $\mathrm{s}^{-1} \mathrm{~cm}^{-2}$

TABLE 9

Gaussian Fits to $\lambda 6$ CM Emission from Feature I

\begin{tabular}{|c|c|c|c|c|}
\hline $\begin{array}{l}\text { Beam } \\
\text { HPBW }\end{array}$ & $\begin{array}{c}\text { Core } \\
S_{\nu}(6 \mathrm{~cm}) \\
(\mathrm{mJy})\end{array}$ & FWHM, PA & $\begin{array}{c}\text { Envelope } \\
S_{\nu}(6 \mathrm{~cm}) \\
(\mathrm{mJy})\end{array}$ & FWHM, PA \\
\hline $\begin{array}{l}2.50^{\prime \prime} \times 2.50^{\prime \prime} \\
2.48^{\prime \prime} \times 1.30^{\prime \prime}\end{array}$ & $\begin{array}{l}2.90 \pm 0.03 \\
2.66 \pm 0.02\end{array}$ & $\begin{array}{l}1.4^{\prime \prime} \times 1.0^{\prime \prime}, 39^{\circ} \\
1.2^{\prime \prime} \times 0.8^{\prime \prime}, 43^{\circ}\end{array}$ & $\begin{array}{l}1.69 \pm 0.05 \\
2.00 \pm 0.07\end{array}$ & $\begin{array}{l}5.6^{\prime \prime} \times 1.9^{\prime \prime}, 142^{\circ} \\
5.5^{\prime \prime} \times 2.6^{\prime \prime}, 145^{\circ}\end{array}$ \\
\hline
\end{tabular}

